LA-U17 -86-1116

$$
\text { CONF-85 } 11170--2
$$

LA-UR--86-1116

DE86 010161

TITLE: MONTE CARLO RENORMALIZATION GROUP: A REVIEW

AUTHOA(S) Rajan Gupta

SUBMITTED :O Vrocecdings of 1985 Wuppertal Conference on: LATTICE GAUCE THEOKIES -- A CHALLENGE IN LARGE SCALE COMPUTING

\title{
DISX'I AIMER
}

This reporl was prepared a. rn eccounl of work sponmored by 1 agency of the I/nited Sluien (iovernment. Neither the (Ini ed Sisten (ivivernment mor any aserity theres), llir any of their employeen, makes any wurrunt /. express or implic il, of assumea any legul liubility or responsibility for the 'occurncy, compi tenew, of unefulnew of any information. appiral:i... nroduct, or process dimeloistl, or represents that its une wouls nol infringe privniely uwnal rignth. Refer-

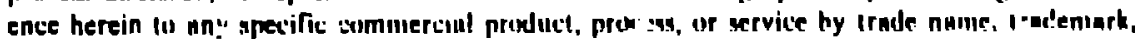
manufaclurer, or wherwise deren not necrsasily crinstilute or imply its endorsement, recum.

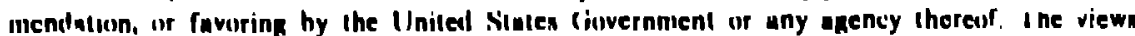

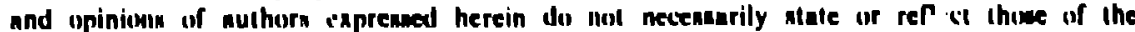
Inlied istex (ioverninent or any apency therail

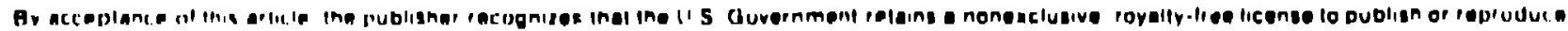

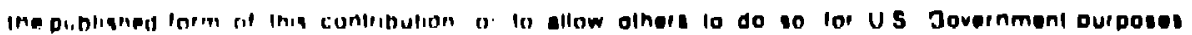




\title{
MONTE CARLO RENORMALIZATION GROUP: A REVIEW
}

\author{
Rujan Gupta† \\ MS-B276, Los Alamos National I,aboratory \\ Los Alamos, N.M. 87545
}

\begin{abstract}
'The logic and the methods of Monte Carlo Renormalization Group (MCRG) are reviewed. A status report of results for 4-dimensional lattice gauge ti:eories derived using $M C R G$ is presented. Existing methods for calculating the improved action are ieviewed and evaluated. The Gupta-Cordery improved $M C R G$ method is described and compared with the stasidard one.
\end{abstract}

The development of Monte Carlo Renormalization group method ( $M C R G$ ) was essentially complete in 1979 with the work of Wilson ${ }^{1}$, Swendsen ${ }^{2}$ ard Shenker and Tobochniks. Prior to this $\mathrm{Ma}^{4}$ and Kadanoff had provided key ingredients. The method is therefore relatively new, furthermore $i$ is application to field theories hai been carried out only since 1982 . In this short period there has been considerable activity and I shall seview the methodology and summarize the status wiih emphasis on 4-dimensional gauge theories. There already exists extensive literat'sre (nn $M C R G$ and I direct the reader to it $1,3,0,7$ for details and fo: a wider exponure. Similarly, the reviews, ${ }^{8,0}$ are a good starting point for beckground on Lattice Geuge Theories and on upin systems. The topics I shall cover are

1) Introduction to $M C R G$ and its methodology.

2) Kenormalization Group Transformations fc: $d=4$ lattice gauge theories.

3) $U(1)$ Lattice Gauge theory.

1) $\beta$-function and Scaling for SU(3) Lattice Gauge Theory.

5) Improved Artions and Methods to calculate them.

6) Improved Monte Carlo Renormalization Group.

7) Effective Field Theories.

The main result in QCD srom $M C R G$ are the determination of the $\beta$-function and the consequent prediction for the value of the coupling at which asjmptotic acaling aet in and second an eatimaie of the improved gauge action ${ }^{10}$. These rewult are not ipectacu!ns in the rense of confrming that QCD is the correct theory of otrong interachloas, however they have led to a deeper understanding of the lattice theory and provided a quantitative estimate of the approach to the continuum limit. I ahall attempt to show that this method is as yet in Ito infancy and should be uaed to tackle a number of problems.

I Invited Talk glven at the Nov. 1086 Wuppertal Conference on: Latlice Gauge Theories - A Challonge in Large Scale Computing.

$\dagger J$. Robert Oppenheimer Fellow 


\section{1) INTRODUCTION TO $M C R G$}

Renormaliention Group $11,12,13$ (RG) is a general framewcrk for studying oystems near their critical point where singularities in thermodynamic functions arise from coherence at all length scales. This phenomenon occurs in Statistical Mechanics near and on the critical surface (defined $t_{y}$ a divergent correlation length) and in the strong interactions of quarke and sluons. The $M C R G$ method was developei to handle this problem of infinitely many coup!ed degrees of ti eedom so that serisible results can be obtained from finite computers. There are two central ideas behind MCRG: One is ton nverage aver theee infinitely many degrees of freedom in discreet oteps preserving only those which are relevant for the description of the physical quantities of interest. The interaction between these averaged (block) fieldo is described by an infinite wet of couplings that get renormalized at each step. In QCD this discrete reduction is sarried out until the correlation length is small enough so that the system can be simulated on a lattice with control over finite size effects. The recond is that thare are no singularities in the coupling constant space even though the correletion length diverges on the critical surface and that the fixed poirt is ahort ranged. Thus even though there are an infinite number of couplings generated under renormalization, only a few ahort range ones are neressary to simulaie the system at a given ucale and preserve the long distance physics. Prejent results suggest that the fixed point for QCD is short ranged.

Standard Monte Carlo: Consider a magnetic nystem consisting of opins $\{s\}$ $n$ nthe sites of a $d$-dimersaional lattice $L$ described by a Hamiltonian $H$ with all possible couplings $\left\{K_{a}\right\}$. All thermodynamic quantities can be found from a detailed knowledge of the partition function

$$
Z=\sum e^{-H}=\sum e^{x_{-} s .}
$$

where $S_{a}$ are the interactions. In Monte Carlo, configurations of apins on the ori inal lattice are generated by the Metropolis 14 , heat bath ${ }^{18}$, molecular dynamics aliac Microcanonical ${ }^{10}$ or the Langevin ${ }^{17,10}$ algorithm with a Boltzmann distribution $e^{-\| I} \equiv e^{K . S .}$. All thermodynamic quantities are given as almple averages of correlation functione over these weighted confgurations. Tile accuracy of the calcuiatione depend on the size of the otatistical sample and the lattice aize $L$ used. Both these quontities depend on the largest correlation length $\xi$ in the ayotem. Near the critical temperature, $T_{c}$, asociated with ancond order phase transitions, the correlation length and consequently thermodynamic quantitien like the npecilic heat elc diverge as functions of $\left(T-T_{c}\right)$ with universal ritical exponents that have been calculeted for many syateras elther analytically or by the Monte-Carlo / MCRG method. Because of a diverging $\xi$, long runs are needed to counter the critical slowing down and the lattice aise has to maintained at a fow timcs $\xi$. The problem of critical alow. ing down to addresed by anriyzing update algorithmn (Mctropolin ve. heat bath vn. Microcanonical vs. Langevin with acceleration techniques like mult/-grid ${ }^{10}$, fourier acceleration ${ }^{10,20}$ etc). The optimum method lo, of courne, nodel dependent and han to take care of metantability (local veraus gloha minima) and global excitationa llke vortices, inatantone otc that are not efficiently handlod by local changes. This last feacure has not recelved adequate attention. To control the sccond problero in atandord Monte Corlo, efferls of a finite lattice especially as $\varepsilon \rightarrow \infty$, finlto size uraling 
has been wed with euccess. In this review I shall concentrate on $M C$ L.G. First I. shall jescribe how universality and caling are explained by the renormalisation group.

The rencrmalization group transiormation $(R G T) B^{1}=R(H)$ is an operator detined on the space of coupling constants, $\left\{K_{a}\right\}$. In practice the $R G T$ is a prescription to average spins over a region of size $b$ the scale faitcr of the $R G T$, to produce the block spin which interacts with an effective theory $B^{1}$. The twc theories $E^{\prime}$ and $H^{1}$ dencribe the same long distance physics but the correlation length in lattice units $\xi \rightarrow \xi$. If this $R G T$ has a fxed poin: $H^{*}$ such that $E^{*}=R\left(H^{*}\right)$, then clearly the theory is scale invariant there and $\xi$ is either 0 or $\infty$. An example of a fixed point with $\xi=0$ is $T=\infty$ and these are triviad. The interesting esee is $\xi=\approx$ sbout which the theory is governed by a single scale $\xi$. If thus fxed point is unstable in 1 direction only (this direction is called the Renormalized Trajectory $(R T)$ ), then non-critical $B$ wili flow away from $B^{*}$ along trajectories that arymptotically converge to the $R T$. Thus the long distance physics of all the trajectories that converge is identical and is controlled by the $R T$. Similarly, points $\epsilon$ away from $H^{\bullet}$ on the $\infty-1$ dimension hypersurface at which $\xi=\infty$ (the critical aurface) will converne to $H^{\bullet}$. The fact that the fxed point with its associated $R T$ control the behavior of $a$ ll $B$ in the neighborhood of $H^{\circ}$ is univessality. Next, cunsider a non-critical $H$ that approaches $H^{*}$ along the $R T$. Thermodynaric ,iuantities depend on a sing'e variable i.e. distance along the $R T$. This is acaling. Corrections to scaling occur when $H$ does not lie on the $R T$. These are governed by the irrelevant eigenvalues of the $R G T$ which give the rate of flow along the critical surfare towards $H^{*}$ and for $H$ not on the $R T$, the rate of convergence towards it. The relevant eige zvalue gives the rate of tow away from the fxed point (along the unstablig direction $R T$ ) and is related to the critical exponent $\nu$. This terse expose ends with a word of caution; all these statements bave validity close to $H^{\bullet}$.

In the $M C R G$ method, configurations are generated with the Boltzmann factor $e^{K . S .}$ as in standard Monte Carlc. The $R G T, P\left(0^{\perp}, 0\right)$, is a preacripticn for averaging variables over a cell of dimension $b$. The blocked variablis $\left\{a^{d}\right\}$ are defined on the sites of a sublattice $L^{1}$ with lattice epacing $b$ times that of $L$. They interact with undetermined couplings $K_{u}^{1}$, however the configurations are distributed according to the correct Boltzmann factor $e^{\prime} H^{\prime}$ i.e.

$$
e^{-H^{\prime}\left(0^{\prime}\right)}=\sum P\left(0^{1}, 0\right) e^{-H(0)}
$$

un expectation values can be calculated en nimple averages. The $R G T$ should satisfy the Kalanoff eruatraint

$$
\sum^{1} P\left(a^{1}, a\right)=1
$$

independent of the statr. $\{0\}$. Thin guaruntes that the 'wo theories $Y$ and $H^{1}$ have the same pertition function. The blocking is done $n$ times to produce confgurations with hamiltunlars $H^{n}$ deocilbing the anme long distancs phyaica but on increasingly cosreer intticos. The fixed point $H^{*}$, the $R T^{\prime}$ id the nequence of theories, $H^{n}$, generated from a given atertirg $H$ depend on the $R G T$. May' different $R S T$ can be used to unalyze a given model (determine th " univeraal exponents) and I defer diacusalon on how to evaluate their efficiency to cectlose 2 and $b$. 


\section{1) Methods to caltulate the critica! exponents.}

There are two methods to calculare the critical exponents from expectation values calculated as simple averages over configurations. In both there is an implicit assumptior that the sequence $H^{n}$ stays cluse to $H^{*}$. The more popular method is due to Swendsen ${ }^{2,7}$ in which the critical exponents are calculated from the eigenvalues of the linearized transformation watrix $T_{\alpha \beta}^{n}$ which is defined as

$$
T_{a \beta}^{n}=\frac{\partial K_{a}^{n}}{\partial K_{\beta}^{n-1}}=\frac{\partial K_{a}^{n}}{\partial\left\langle S_{\sigma}^{n}\right\rangle} \frac{\partial\left\langle S_{\sigma}^{n}\right\rangle}{\partial K_{\beta}^{n-1}} .
$$

Each of the two terms on the right is a connected 2-point correlation function

$$
\frac{\partial\left\langle S_{\sigma}^{n}\right\rangle}{\partial K_{\rho}^{n-1}}=\left\langle S_{\sigma}^{n} S_{\theta}^{n-1}\right\rangle-\left\langle S_{\sigma}^{n}\right\rangle\left\langle S_{\beta}^{n-1}\right\rangle .
$$

and

$$
\frac{\partial\left\langle S_{\sigma}^{n}\right\rangle}{\partial K_{\beta}^{n}}=\left\langle S_{\sigma}^{n} S_{\beta}^{n}\right\rangle-\left\langle S_{\sigma}^{n}\right\rangle\left\langle S_{\beta}^{n}\right\rangle .
$$

Here $\left\langle S_{\sigma}^{n}\right\rangle$ are the expectation vaiues on the $n^{\text {th }}$ renormalized lattice and $K_{\sigma}^{n}$ are the correspording couplings. Th: exponent $\nu$ is found from the leading eigenvalue $\lambda_{t}$ of $T_{a p}^{n}$ as

$$
\nu=\frac{\ln 6}{\ln \lambda_{t}}
$$

where $b$ is the scale factor of the $R G T$. The eigenvalues leas than one give exponenty that control corrections to ecaling. The accuracy of the calculated exponents improves if they are evaluated close to the fxed point. This can be achieved by starting from a critical point and blocking the lattice a sufticient number of times i.e. for large $n$. Thus the convergence is limited by the starting lattice size and how close the starting $H^{c}$ is to $H^{*}$. If $H^{*}$ can be approximated by a small number of short rar.ge couplings (a necessary assumption in the RG), then this method can be inproved if the renormalized couplings $\left\{K^{n}\right\}$ are determined starting from a known critical Hamiltonian. These should then be used in the updale. A second possibility is to tune the $R G T$ oo that the convergence to $H^{*}$ from a starting $H^{c}$ is improyed. In section 5, I will dencribe a number of methods to calculate the renormalizc colplings. Tuning of the $R G T$ is discussed in section 2.3 and a careful analysis of the accuracy of this method is deferred until section 6 .

The second method to calculat a the leading relevant exponent is due to Wilson" Consider once again the 2-point connected correlation function (the derivative of $n$. expectation value) $\left\langle S_{a}^{i} S_{p}^{j}\right\rangle_{c}$ with $j>i$. Expand $S_{a}^{i}$ in term of the eigenoperators $O_{a}^{i}$ of the $R G T$. Close to $H^{*}$ the level dependence in $O_{a}^{i}$ (equivalently in the expansion coefficients $c_{a p}^{i}$ ) can be neglected. Shen to the leading order

$$
\left\langle S_{a}^{i} S_{\rho}^{j}\right\rangle \sim \lambda_{i}^{j-i} c_{a, i}\left\langle O_{i} S_{\rho}^{j}\right\rangle
$$

where $\lambda_{1}$ is the leading relevant eigonvalue and corrections are auppreased by $\left(\lambda_{1}\right)^{j-i}$.

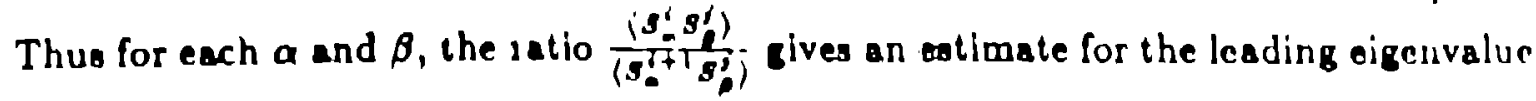


$\lambda_{t}$. The accuracy of the method improven if $j-i$ is large since non-leading terms are suppressed geumetrically. Sc far this method has not been used extensively so its practical accuracy cannot be evaluated.

QCD: At the tree level, the coupling $g$ in QCD does not renormalize and the fixed point is at $g_{\text {bare }}=0$. At 1-loop the leading operator has eigenvalue equal to one, is relevant and the fixed point changes from simple gaussian to being asymptotically free and non-trivial. A special feature of asymptotic freedom is that even when the leading eigenvalue is one there is a flow away from the fixed point at a constant rate. At 2-loop, this operator becomes truely relevant i.e. with eigenvalue $>1$. Perturbution theory also tells us that leading eceling vicletions are $\sim \frac{1}{k^{2}}$, so the second eigenvalue should be $\sim \frac{1}{b}$ for a $R G \Gamma$ with sca'e factor $b$. Present studies $23 b$ show that the leading eigenvalue is clase to 1 and the second newr $\frac{1}{b^{2}}$. However, the statistics are poor and the calculation was done at large gbare. Thus reliable quantitative results are lacking.

\section{2: Wllson's method to fiud a critical polnt}

Consider $M C R G$ aimulations $L$ and $S$ with the same starting couplings $K_{a}^{0}$ but on lattice sizes $L=b^{n}$ and $S=b^{n-1}$. If $K_{a}^{0}$ is critical and after a few blockings the 2 theories are clooe to $H^{*}$, then all correlation functions at tain their fuxed point values. For non-riticai starting $H$, expand abcut $H^{*}$ in the linear approximation

$$
\begin{gathered}
\left\langle L_{a}^{m}\right\rangle-\left\langle S_{a}^{m-1}\right\rangle=\frac{\partial}{\partial K_{\beta}^{0}}\left\{\left\langle L_{a}^{m}\right\rangle-\left\langle S_{a}^{m-1}\right\rangle\right\} \Delta K_{\beta}^{0} \\
=\left\{\left\langle L_{a}^{m} L_{\beta}^{0}\right\rangle_{c}-\left\langle S_{\alpha}^{m-1} S_{\beta}^{0}\right\rangle_{c}\right\} \Delta K_{\beta}^{0}
\end{gathered}
$$

to determinc $\Delta K_{a}^{n}$. To reduce finite size effects the compared expectation values are calculated on the same size lattices. The critical coupling is given by

$$
K_{a}^{c}=K_{a}^{0}-\Delta K_{a}^{0}
$$

and this estimate should be improved iteratively.

$C_{n}$ the critical surface the 2-point corre!ation functions (like in Eq. (1.5) and (1.A)) divergc in the thermodynamic limit. However, their ratio is the rate of change of coupliıgs ana these ase well behaved. The reason MCRG has better contiul over finite size effects is that if $H^{*}$ is short ranged then only short ranged correlation functions need to be evaluated in determining $T_{a \rho}^{n}$ or in Eq. (1.9). The finite aize contributions to the ratios fall off like the couplings i.e. exponentially. Thus reliablc estimales are obtained from omall lattices.

\section{2: RENORMALIZATION GROUP TRANSFORMATIONS IN $d=4$}

It has been mentioned before that there is no unique $R G T$ for a given model. There are at present four different tranoformations that have been proposed for 4-dimensional lattice gauge theories. In each of them the block link variable is conetructed from a sum of paths $\Sigma \equiv \sum$ paths. Thls sum of SU(N) niatrices 


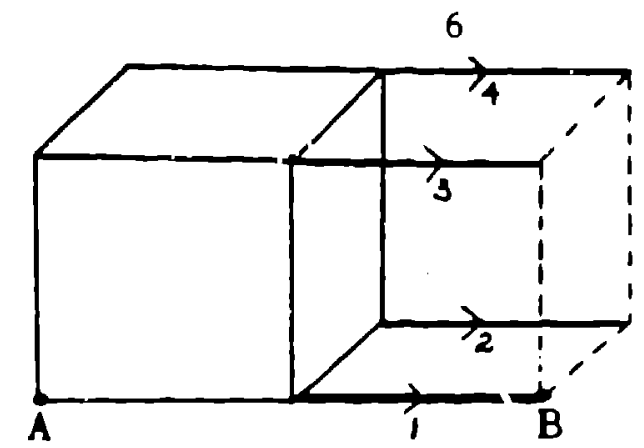

Figure 1: Wilson's b - 2 RCT. Four of the elght paths in 8 given direction are nown.

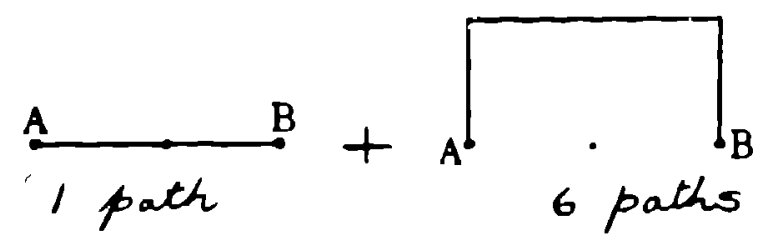

F1gute 2a: Swendsen's b- 2 R?T.

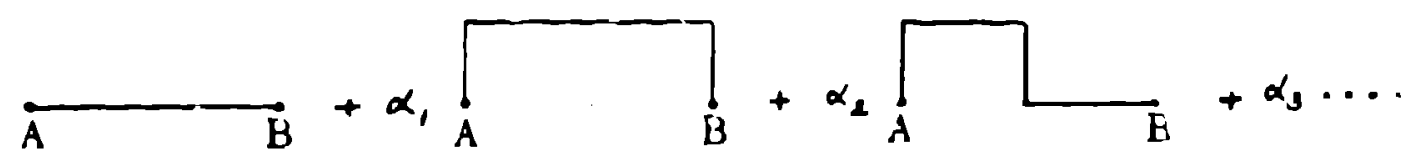

Figure 2b: Generalized Swendsen RCT with parapeters $\alpha_{i}$ that hava to be optimized.

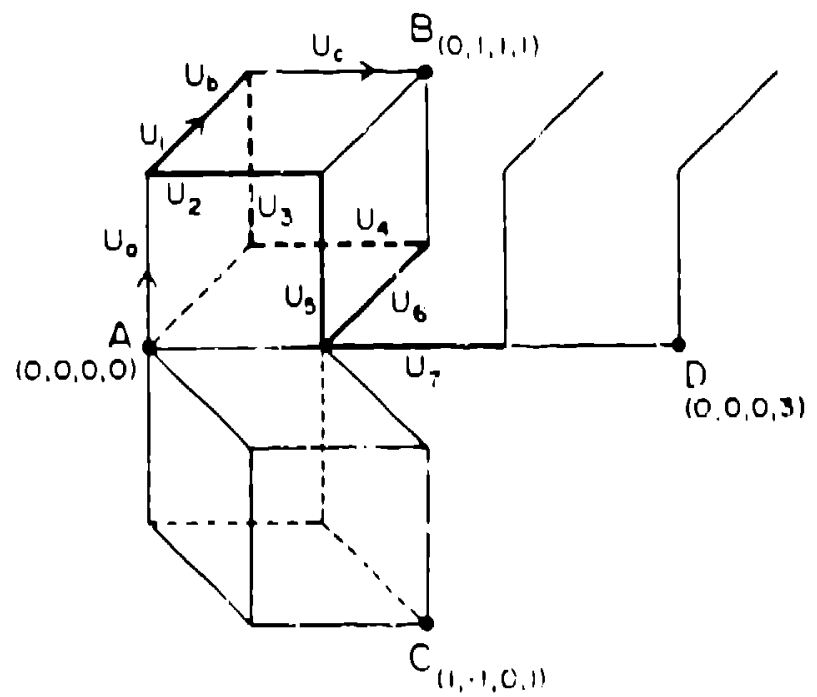

4-DIMENSIONAL HYPERCIÜIC LATTICE

F1guris 3: The gecmetry of the $\sqrt{3}$ block tranaformation. 
is noi an element of $S U(N)$, and the new block mix matrix is selculed with iie. distribution

$$
P\left(U_{b}\right)=e^{P \operatorname{Tr} U_{b} \Sigma}
$$

where $p$ is a free parameter to be optimized. The advantage of taking the sum is that such a $R G T$ preserves gauge invariance. The $4 R G T$ are (in cronological order)

2.1) $b=2$ by Wlsor ${ }^{1}$ : The geometry of the transformotion is shown in Fig. 1. There are 8 links in a given directior. of which 4 are shown in the 3-dimensional projertion. In this method the gauge has to be fixed on 15 siten other than the block site. This fixing has to take into account the fact that she ends of the 8 links are ai different sites. The ansaiz Wilson used was to tranaform. the hypercube locally into the Landau gauge. The process of fixing the gauge in slow and $\%$ disadrantage of the method. The need for gauge fi: ing can be avoided by defining 8 paths that run between the block sites and include the same links. This modified construction violates cubic rotational invariance because of the particular choice of the ordering of the paths within the cell. In both forms only $\frac{92}{10}$ degrees of freedom are used in this approximate averaging at each level. This method has not been used sirice Wilson's preliminary investigation because the next two methods are simplut.

2.') $b=2$ by Swendsen ${ }^{21}$ : The transformation in its initial form is shown in Fig. 2a. The more general version is shown in Fig. $2 b$ where the parameters $a_{i}$ have to be determined. In this constructicn all paths start and end at the block sites. ihus nu gauge fixing is necessary and arbitrarily complex paths can be included. However calculations show that an optimization of the parameters has to be done to iwprove the corvergence. I shall discuss this cunirg later.

2.3) $b:=\sqrt{3}$ by Cordery, Gupta and Novotny ${ }^{2 z}$ : This trassformation is specific to gauge theories in 4-dimensions and is based on the fact that the body diagonals of the 4 positive 3 -cubes out of a site are orthogonal and of length $\sqrt{3}$. The geometry is shown in Fig. 3 and under one $R G T$ the now lattice in still hypercubic but rotated with respect to the olt basis. Also, the box boundary becomes jarced. This can be undonc by a second application of the $R G T$ with different basis vectors. So the original box geometry is recovered after every scale change by a factor of 3 . The construction of the puths requires no gauge fixing, all paths ars of equal length (no free parameters to be tuned) and 31 degrees of freedom are ured at each step. Further, the block cell consists of the block site and its 8 nersest neighbors. This provides ar. easy and natural way to include complex matter flelds and block them inmultaneously. It is also beiter suited to the fermion block diagonajization pi ccess of Muttnr and Schilling ${ }^{0+}$ as explained in section 5.10. In practice, for both $\mathrm{SL}^{\prime}(2)$ and $\mathrm{SU}(3)$, this $R G T$ has consistently shown good convergence at strong and at weak coupling. It is therefore recommended.

2.4) $b=\sqrt{2}$ by Callaway and Petronzlozs: The construction of Faths shown in Fig $4 a$ in bas $s$ on a planer atructure i.e. $x-y$ and $z$-t planes are treated scparately at all blocking steps. No gauge flxing is required but only 2 paths are used in the averaging i.e, lin Eq. (2.1). This drawback of using only 2 planar paths can be improved by law lisllng nonplanar pailo an shown in Fig. 1b. Bccause this $R G I$ hos tho advantage that $b=\sqrt{2}$ is the omallest acale factor possible, a serious test should be mode. 


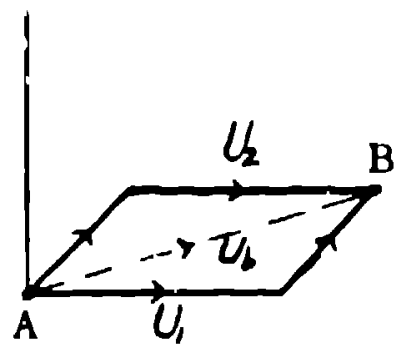

Figure 4a: The two paths in the b $=\sqrt{2}$ RGT.

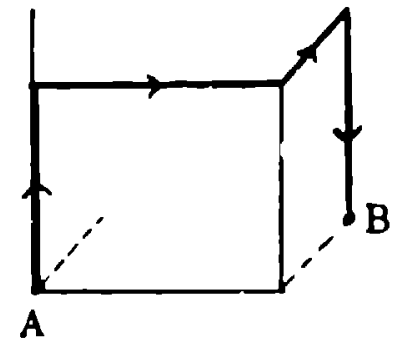

Figure 4b: Additlonal 4 link paths.

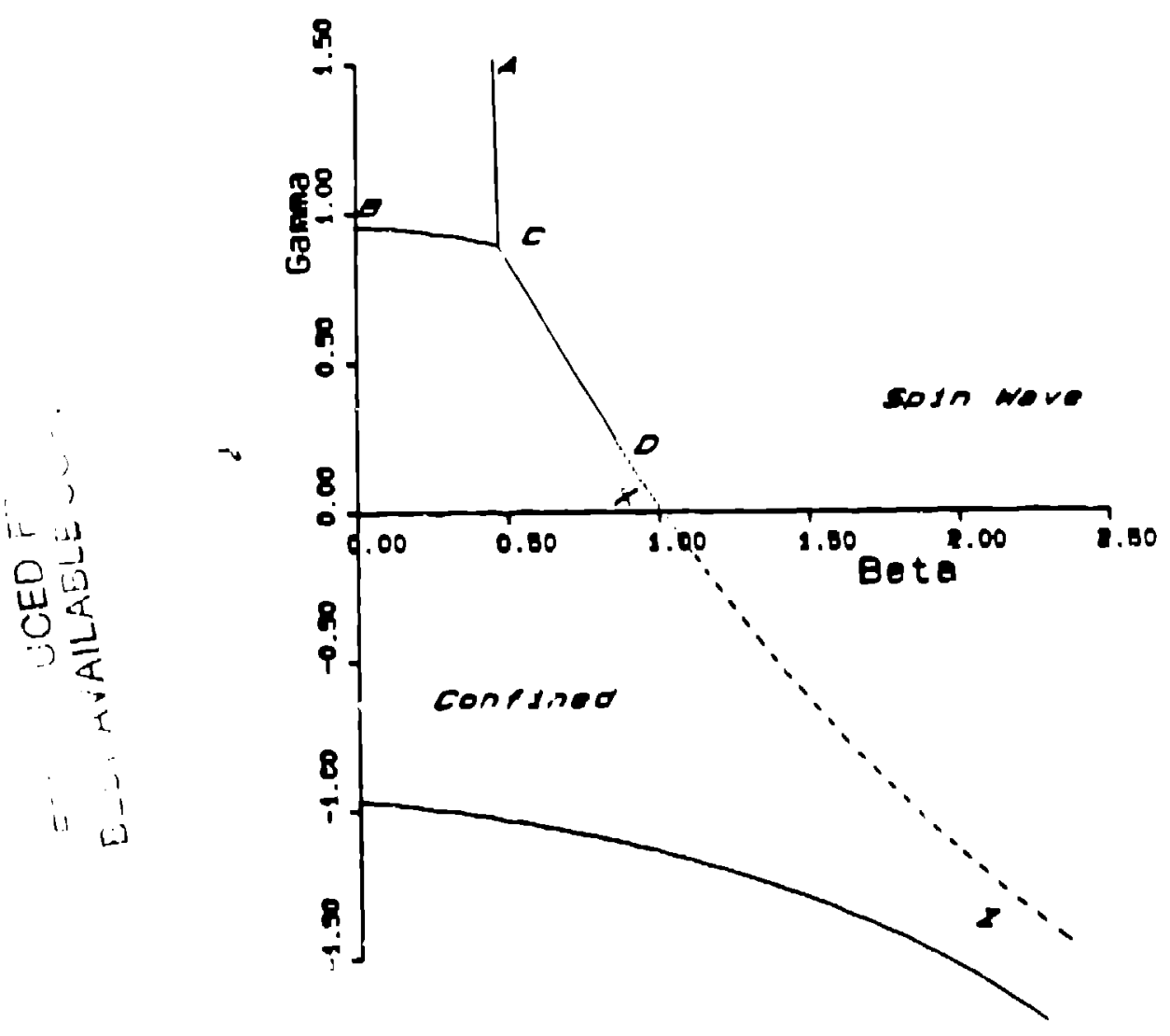

Figure 5: The phase Jlagram of U(1) gauge theory. The location of the TCP 1. mewhere near the dotted line DX. 
2.5) Optimieation of the RGT: In addition to the freedom of the choice of the $K G T$, there are the free parameters $p$ and $\alpha_{i}$. Hasenfratz et al. ${ }^{24}$ have shown that the convergence of the original $b=2$ Swenduen trassformation is improved if $p$ is tuned. I will give a qualitative description of how this works. Considar a set of $R G T$ that are a function of the continuous parameter $p$ i.e. $R_{p}$. Starting from a given point $B$, the $b^{-}$. xked theories generated are described by $B^{1}(p)$. They all have the same bng diatance behavint as can be checked by measuring expectation values of large Wilgon loops. In fact there is an effective Wibon action $\boldsymbol{H}$. /s which will have the same loug distance behavior. The short distance behavior of $H(p)$ will be different and for some values of $p$, the (plaq $\rangle_{p}$ will be larger than the (plag) corresponding to $H_{\text {eff }}$. I have checked that this is the case for the criginal Swendsen transformation when $p=\infty$ and $g^{2}<1$. Lowering $p$ reduces the blocked $\langle p l a q\rangle_{p}$, making it agree better with $B_{\text {efs. The }}$ The the tuning makes the short and long distance behavior correspond better to the same approximate $H_{\text {af } f \text {. This improves }}$ the nacching (using small loops) in the 2-lattice method to calculare ine $\beta$-function. Hamenfratz et al. ${ }^{24}$ estimate $p$ using perturbation theory and by Monte Carl, using the criterion of early matching of tlock expectation values in Wilynn's two lattice methed. They found that the best velue at $\frac{\theta}{g^{2}}=6$. given by Monte Carlo $(\sim 35)$ does not agree with the value found using perturbation theory $(\sim 15)$. So as of now this optimization is still by trial. 1 lso, $p_{\text {opt }}$ depends on the coupling $g$. This implies that the $R T$ cannot be pulled close to the Wilson axis globally by this optimiration. So the usefulness of such optimization is limited to the $\beta$-function calculation. The parameters $\alpha_{i}$ can similasly be optimized using the same improvement criverion.

Gupta and Patel ${ }^{22}$ used $p=\infty$ in the $\sqrt{3} R G T$. This is equivalent to choosing the matrix $U$ such that $\operatorname{Tr} U \sum$ is waximized (the $\delta$-function construction). They find that even with this choice the omall block Wilson loops are more discrdered than for an $H_{\text {a }} /$ determined using large loops. Thus lowering $p$ would not help. The $\sqrt{3} R G T$ has shown good convergence properties and provided reliable results with $p=\infty$.

The freedom to choose the $R G T$ and further tune the parameters $\alpha_{i}$ and $p$ leads to the question: What are the criteria by which to decide what is the best $R G T$ ? I will first address the question - - what is the effect of chinging the $R G T$ on the fixed point and on the RT? Conjecture ${ }^{28}$ : Changing the RCT inoves the fixed point on the critical surface but only along redundant directions. A simple argument is as follows: Consider two different $R G T, R_{1}$ and $R_{2}$, and their associated fixed points $H_{1}^{*}$ and $H_{2}^{*}$. There are no non-analytic corrections to ocuing at either fixed points and the associated $R T$. If these two points are distinct, then under $R_{1} H_{2}^{*}$ flows to $H_{1}^{*}$. Consequently there are no ocaling violations along the flow. This is by definition a redundant direction. This implies that the associated $R T$ differ by redundant operators.

The presence of redundant operators does not effect the physics, however it can obscure resulto. 'The redundant eigenvalues al a not physical, depend on the RGT, and can be relevant or irrelevant. If a relevant redundant operator is present then the flows will not converge to the $H^{*}$ or to the $R T$. Thus it is desirable to pick a $R G T$ for which the redundant oigenvalues are amall. Similarly, the coeficients of the leading irrelevant operaton should be reouced. To some extent the irrelevant basis vectors are a function of the position of $H^{*}$, wo it $\mathrm{L}$ possible to simultaneousiy reduce the two coefficients. In $Q C D$. there is an additional freedom - - all possible 
Wilson loops form on overcomplete set. Therefore, in order to tune the $R G T$ and to find an efficient improved action, it is necessary to determine the operators that can be eliminated because of the overcomplateness - nd the redundant combinations.

Swendsen ${ }^{20}$ has conjectured tinat the fixed point can be moved anywhere un the critical surface by tuning the $R G T$. In part:ailar, if the simulation point is made $H^{*}$, then that $R G T$ is optimal. There is some support for this in spin systems, where by adding terms to the $R G T$, one can successively kill terms in the renormalized hamiitonian. There are two things to check here: first whether the coefficienty of the $R G T$ terms fall off like thr couplings, i.e. exponentially, and second whether the lone range untuned couplings continue to fall off at least as fast as before. The qrantity to optimize is the update complexity (embodied in the $R G T$ or the hamiltonian) virsus the decrease in the coefficient of the leading irrelevant operator. Swendsen ${ }^{26}$ found that the eigenvalues for the $d=3$ Ising model are significantly improved with a tuned 10 term $R G T$. However, he did not compare it with a simulation that used a 10 term truncated renormalized hamiltonian close to the $H^{*}$ for a simple $R G T$. There is one additional anomaly in this approach: Tuning the $R G T$ improved the thermal exponent but the results fo: the magnetic exponent deteriorated in quality. This is surpriving because the fixed point is at zero odd couplings and these remain unchanged in tuning the $R G T$. The previous conjectures are in conflict and the results are ambiguous. Consequently, this subject is being explured ${ }^{60}$ further.

The criterion for an optimum $R G T$ is to make the $H^{*}$ and the $R T$ as short rarged as possible. In critical phenomena, the improvement can be quantified by measuring ithe convergence of the exponents as a function of the blocising level. In $Q C D$ we are interested in continuum mass-ratios etc. These have so far been hard to measure so the improvement cannot be judged. The behavior of the $R T$ for $Q C D$ ii diac ussed at the end of section 5. For the moment let me conclude this section by: The the question of how best to optimize $M C R G$ has not been adequately answered and is under investigaticn.

\section{3: U(1) LATTICE GAUGE THEORY:}

The phase diagram of the theory defined by the action

$$
S=\rho \sum \cos \theta_{\mu \nu}+\gamma \sum \cos 2 \theta_{\mu \nu}
$$

where $\beta(\gamma)$ is the charge 1 (cherge 2) coupling is known to have 2 phase boundary separating the confining (sirong-coupling) phase from the spin-wave (QED) phase ${ }^{27,28,20}$. The order of the transition along the boundary $D X Z$ in Fig. 5 is not known. In partic ilar it is not known if the gradually weakening first order transition along $C D$ ends in a tricritical point, and if so what is its location. Evertz et al ${ }^{28}$ claim that the location of the $T C P$ is at $\beta=1.06 . t 0.04$ and $\gamma=-0.11 \pm 0.05$ on besis of a ocaling analysis of the discontinuity in the energy $\Delta E$. 'The mechanism driving the transition are topological excitations ${ }^{30,31}$, i.e. closed loor 3 of raonopoles, whose density is observed to change at the transition ${ }^{32,33}$. This change in density is caused by arowth in the size of the largest monopole loop which begins to span the finite lattices used in the calculations ${ }^{32,34}$. Thus, the usual difficulty of inite size effects near a TCP in determining the loration of the TCP by ar extrapolation 
of the latent heat $\Delta E$ along the phase boundary is here compounded by the presence of monopole current loops that are closed due to the lattice periodicity ${ }^{32,34}$. These contribute a fake piece to the $\Delta E$ winich makes the extrapclation unreliable. One solution is to calculate and then subtract the contribution of these loops from $\triangle E$ before making the extrapolation. The more reliable method is $M C R G$ and in particular the 2-lattice method discussed in section 1.2 should be used to locate the TCP. A word of caution for the U(1) model when using this method: There is a large shift in the critical coupling as a function of the !attice size ${ }^{32}$ and consequently in the contribution of the fake monopole loops. One should therefore use a starting coupling for which both lattice simulationa are on the same side of the trannition?

The present status is that in a $M C R G$ calculation done along the Wilson axis ${ }^{32}$

only one relevent exponent was found using the $\sqrt{3} R G T$. Furthermore, the value of the exponent showed a variation with $\beta$. At $\beta=1.0075, \nu \approx 0.32$ and this value changes to $\nu \approx 0.43$ (or even the classical value 0.5 ) at $\beta=1.01$. One explanation is that the TCP lies above the Wilson axis and in simulations along the Wilsor. axis one first measures the tricritical exponent and then the critical one. The same conclusion is also reached in a recent $b=2 M C R G$ study ${ }^{3 B}$. Therefore the location of the TCP is still an open question.

The interest in this model (goal of MC calcuiations) is to know if there exists a ixed point at which a non-trivial field theory can be defined. To settle this important question requires considerably more work.

\section{4: $\beta$-FUNCTION AND SCALING FOR SU(3) LATTICE GAUGE THEORY}

The non-perturbative $\beta$-function tells us how the lattice spacing goes to zero as $g_{\text {bare }} \rightarrow 0$. Since on the lattice all dimensionful quantities, like masses, are measured in units of the lattice spacing $a$, we need to know how a scales in order to take the continuum limit. One option is to use the 2-loop perturbative result provided it is demonstrated that this is valid at values of goar. where the calculations are done. The other is to measure the non-perturbative $\beta$-function. In case there is only asymptotic acaling, this calculation is still necessary since it provides the value of goar. at which such scaling setn in.

There are twu methods for culculating the non perturibetive $\beta$-function directly.

4.1) MCRG using Wilson's 2 lattice method ${ }^{1,3}$ : There are 2 groups who have used this mathod for $\mathrm{SU}(3)$; one with $b=\sqrt{3} R G T^{97}$ ind the econd ${ }^{24}$ with $b=2$ proponed by Swend $\Lambda^{21}$. The outline of the method is: First a system of size $L=\left(b^{n}\right)^{d}$ is simulated $w^{i} c h$ couplings $K_{a}^{A}$ and the expectation values of Wilson loops are calculated on the original lattice and the $n$ block lat tices. A second syatem of size $S=\left(b^{n-1}\right)^{d}$ is then simulated with couplings $K_{a}^{B}$ (chusen judiciously) and again the expectation values are calculated on the $n$ lattices The expectation values from the two simulation are compared with the ones from the larger lattice $L$ blucked one more time i.e. $L^{m}$ with $S^{m-1}$. Finite size affects are uninimired since the comparison is on approximately the same physical nize lattices. The couplings $K_{a}^{B}$ are adjusted (which requires $n$ new simulation) until there in matching at the last, $n^{\text {th }}$, level. In practice it is sufficient to do two simulations $S_{1}$ and $S_{2}$ which bracket $L$ and 
then use interpolation. The test for convergence of the two theories $L^{m}$ and $S^{m-1}$ is that the expectations values should match simultaneously at the last few levels. This situation is thown in the coupling constant space in Fig. 6. At matching, the correlation length at $K_{a}^{A}$ is larger than at $K_{\alpha}^{B}$ by the scale factor $b$. Thus if the atarting trajectory is taken to be the Wilson axis (or any 1 parameter line) then the value of the $\beta$-function, $\Delta \beta$, for a acale change $b$ is $K^{A}-K^{B}$.

Under the asoumption that the fixed point action is local, and that at any acale a few short range couplings are sufficient to characterize the action, matching the expectation values of a few small Wilson loops is sufficient to guarantee that the two actions are equal. Recall that there is a one to one correspondence between the value of the couplings and the expectation values. Also note that finite size effects in expectation values are irrelevant once there is matching because then the two theories flow along a common trajectory under a $R G T$ and continue to match. Thus it is oufficient to require that matching first take place on lattices which are large enough to accommodate the important couplings. Thereafter, the check can be on a $1^{4}$ lattice tool It is the range of the couplings that controls finite size effects in $M C R G$ and not the correlation length and this range falls off exponentially even on the critical surface. This is why $M C R G$ has better control over finite size effects and is a powerful method.

For the simple plaquette $\mathrm{SU}(3)$ action with $K_{k} \equiv \frac{0}{0^{2}}$, asyopptotic acaling is defined by the 2-loop perturbative $\beta$-function,

$$
\frac{\partial\left(g^{-2}\right)}{\partial(\ln a)}=-\frac{11}{8 \pi^{2}}-\frac{51}{64 \pi^{4}} g^{2}+\cdots .
$$

The quantity calculated using $M C R G$ is,

$$
\Delta \beta=-\frac{\partial\left(6 g^{-2}\right)}{\partial(\ln a)} \cdot \ln b,
$$

i.e., the discrete $\beta$-function at $K_{\boldsymbol{r}}$ evaluated for a acale change $b$.

The results for the $b=\sqrt{3}$ calculation ${ }^{37}$ are shown in Table 1 , while those for $b=2$ are ohown ${ }^{21}$ in 7 able 2 . There is clear evidence of a dip at $\frac{\theta}{p^{2}} \sim 6.0$ which is caused by the end point of the phase transition line in the fundamental-adjoint coupling opace. The conclusion of these calculations is that there is no asymptotic scaling below $\frac{\theta}{0^{2}}=6.1$.

4.2) Loop ratlo method ${ }^{31,24}$; Thin method is oimpler as it uses expectation valuea of Wiloun loops calculated in standerd Monte Carlo. Thus it can be ued for gauge theory with dynamical fermlone while method 4.1 cannot until one learns how to block fermion. The ration of Wibon loope that cancel the perimeter and corner terms

$$
R(i, j, k, l)=\frac{W(k, l)}{W(i, j)} \quad \text { where } i+j=k+l .
$$

antiffy en approximate homozeneous renormalisetion croup oquation

$$
R\left(2 \vdots, 2 j, 2 k, 2 l, g_{0}, 2 L\right)=R\left(i, j, k, l, \rho_{b}, L\right) \text {. }
$$




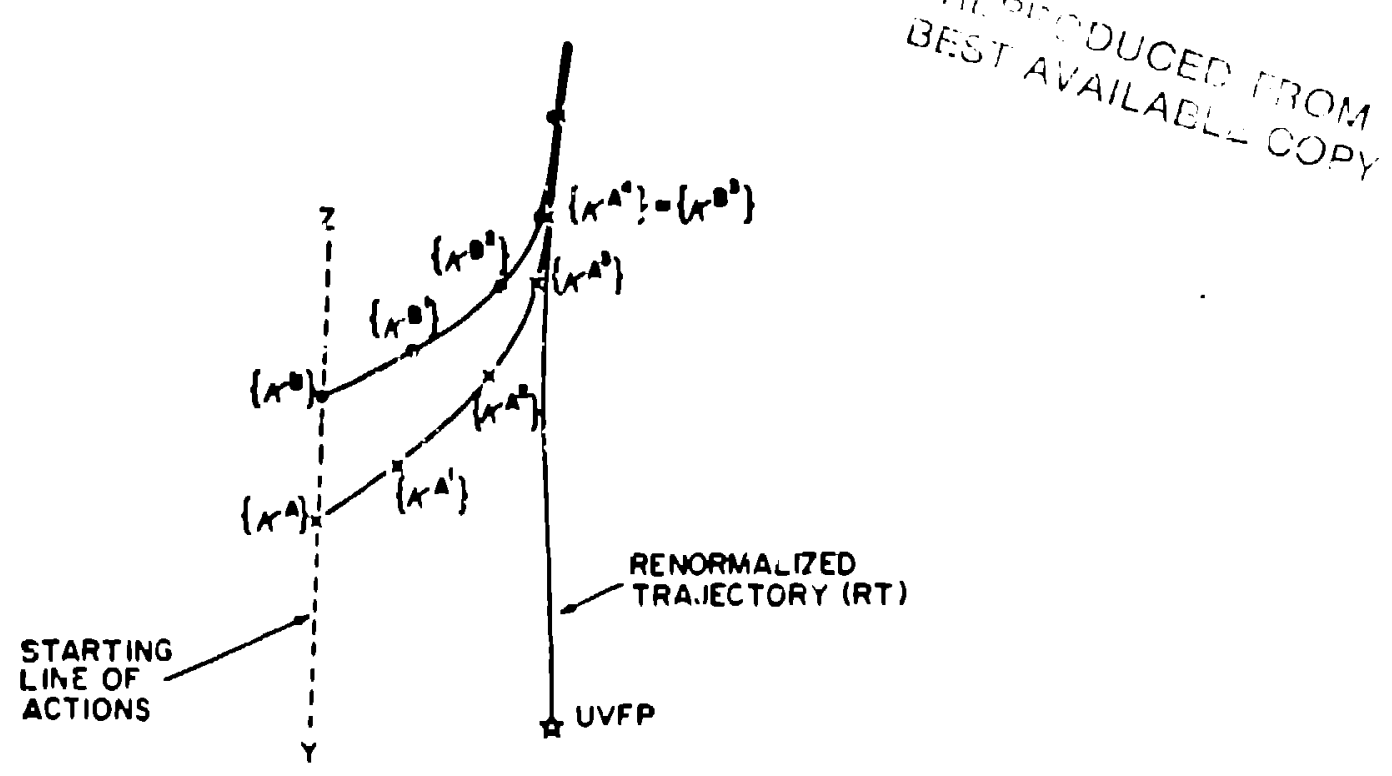

Figure 6: The evolition of actions under the renormalization group traneformatson. The two actions $\left\{K^{A}\right\}$ and $\left\{K^{B}\right\}$ have the uase long-diatance behavior and their lettice correlation lengtho are related by the ocale tranoformation factor $b$.

\begin{tabular}{|l|c|c|c|c|}
\hline \multicolumn{5}{|c|}{$S U(3)$} \\
\hline $9^{4}$ & \multicolumn{3}{|c|}{$\Delta \beta$ for $b=\sqrt{ } 3$ from matching on } & $2-100 \mathrm{p}$ \\
\cline { 2 - 5 }$K_{F}$ & $3^{4}$ & $(\sqrt{3})^{4}$ & $1^{4}$ & $\Delta \rho$ \\
\hline 6.0 & $.337(5)$ & $.323(5)$ & $.308(0)$ & .489 \\
\hline 6.125 & $.387(5)$ & $.376(5)$ & $.351(6)$ & .488 \\
\hline 6.25 & $.421(4)$ & $.424(5)$ & $.401(5)$ & .488 \\
\hline 6.35 & $.431(4)$ & $.452(5)$ & $.445(9)$ & .487 \\
\hline 6.45 & $.432(4)$ & $.464(6)$ & $.425(12)$ & .487 \\
\hline 6.5 & $.435(4)$ & $.464(6)$ & $.449(15)$ & .487 \\
\hline 6.75 & $.430(4)$ & $.485(5)$ & $.443(9)$ & .485 \\
\hline 7.0 & $.422(7)$ & $.503(11)$ & $.488(20)$ & .484 \\
\hline
\end{tabular}

Table 1 : The val "es of $\Delta \beta$ for $b=\sqrt{ } 3$ at diferent levelo of matching for different value of the couplloge " The matching $\boldsymbol{K}_{\boldsymbol{F}}$ on $(3 \sqrt{3})^{4}$ were determlned by linear interpolation and the erron are bacal on a lo Al. Also shown are the values of $\Delta B$ onrresponding to erymphtic scallop. 
Thus using Monte Carlo data for ratios calcrilated on 2 lattices of size $2 L$ and $L$, with couplings $g_{a}$ and $g_{b}$ respectively, the riesired function $\Delta \beta$ for $b=2$ can be calculated. Caveats: Eq. (4.4) becones resrect only as $i \rightarrow \infty$, otherwise there are corrections due to lattice artifacts. The quality of results for large $i, j, \ldots$ are limited by statistics. The reliability of the resultn therefore depends on obtaining the same $\Delta \beta$ fo: $i=1,2,3,4, \ldots .$.

The contribution of lattice artifacts can be reduced in perturbation theory. For this consider Eq. (4.4; for a linear combination of loop ratios with coefficients $a_{i}$. $T u$ determine the $a_{i}$, use the expectations values of loops calculated in perturbation throry and require that $\Delta \beta=0$ (tree-leve!), 0.579 (1-loop) .... Then go back and we the monte carlo data ior Wileon loops to calculate $\Delta \beta$. The drawback of this approach is that if two (or more) ration of different scale, $i=1$ and 1 say, are used then the difference in stailistical errors is a problew. Otherwise, at weak coupling each ratio roughly sativfles $\mathrm{Eq}_{\mathrm{q}}$ (4.4) and there is a lus of sensitivity in determining $\alpha_{i}$. At strong coupling, perturbation theory calculation/improvement of $\alpha_{i}$ breaks down. So one cari, at best, expect a win dow where reliable result are obtained Henenfrats et al. ${ }^{24}$ claim this is true for $\frac{b}{b^{2}}$ in the range $[6,6,6]$. Their results are in ag:eement with their $b=2 M C R G$ resul's as shown in Table 2. It has been observed by Eutbrod ${ }^{30}$ in SU(2) that at ability with respect to loop size is reaciled slowly. Therefore, one has to be cauticus of apparent convergence

4.3) Henslts: It is hard to compara the results of the $b=\sqrt{3}$ study directly with the $b=2$ ones $i$ xause of the different scale factor of the $R G T$. Petcher ${ }^{40}$ has carried out the following aralysis: he fits the $b=\sqrt{3}$ dala to a sooth function which had tha correct asymptotic value built in. This function can then be used to determine the discrete change $\Delta \beta$ in the couplings for any other scale factor $b$. In Fig $?$ the amooth function found from the $\sqrt{3}$ date 1 eacaled to $b=2$ is compared with the $b=2 M C R G$ dace.

Next. we would like to check if the $\Delta \beta$ calculated from MC determinations of different physical observables are identical and agree with the $M C R G$ calculations. This comparison tests two things, first whether there exists acaling (constant mass ratios) before (larger $g$ ) asymptotic acaling and second whether the MC measurements are reliable. The lattice value of a mass ma calculated at two values of the coupling, of and $\frac{f}{p}$, gives the $\Delta \beta$ for acale change $a_{2}$. Unfortunately the values of couplings cannot be selected to give the $\Delta \beta$ for a given constant scale thange. This again introduces the problem of rescaling datu thereby preventing a defnite atstement on acaling. In F'B. 8 wo have only used pairs of data points with a acalc factor close to $\sqrt{3}$. At $\frac{8}{1}=6.0$, the $0^{++}$glueball inese ${ }^{41}$, atring tension $\sigma^{42}$ and the deconfinement temperature $T_{c}{ }^{43}$ represent ocales of 2,5 and 8 lattice unite respecti ely. Thus identical $\Delta \beta$ would be n resoonable test of acnling. Bearing in mind the problem of reacaling data, the only aignificant atatement is that the behavior of the glueball mase is different.

The oneet of asmptotic acaling hw also been checked by plotting wa where $m$ is the deconfinement temperature $T_{a}$ and $\Delta$ ib the 2-loop perturbatlve acale. Kuti et al. ${ }^{4 s}$ found that for $N_{r}=10,12,14$ thi: atio $b$ conatant and different from the value at $N_{r} \leq 8$. From this they deduce that there in aymptotic acaling for $f>6.18$. The only drrivieck of this method to the relience on 1 calculated in 2 loops to define nymr cotic mcallng. There could be correctlons i.e. $\left(1+O\left(\sigma^{2}\right)\right)$ terms, 


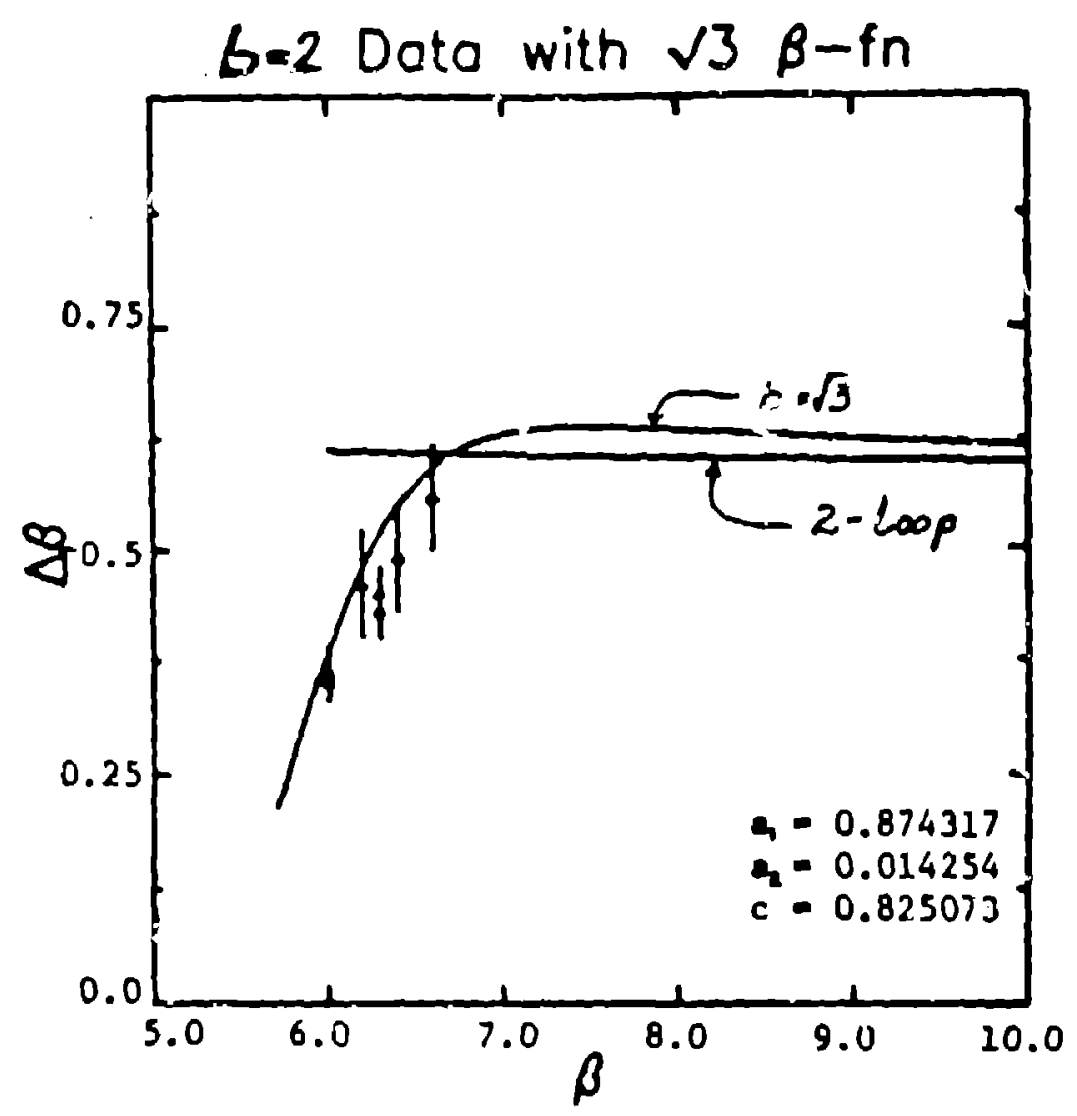

Figure 7: The wooth $c l$ is a itt to the $b-\sqrt{3}$ data. The data pointe are for b -2 , (Courtesy of D. Petcher)

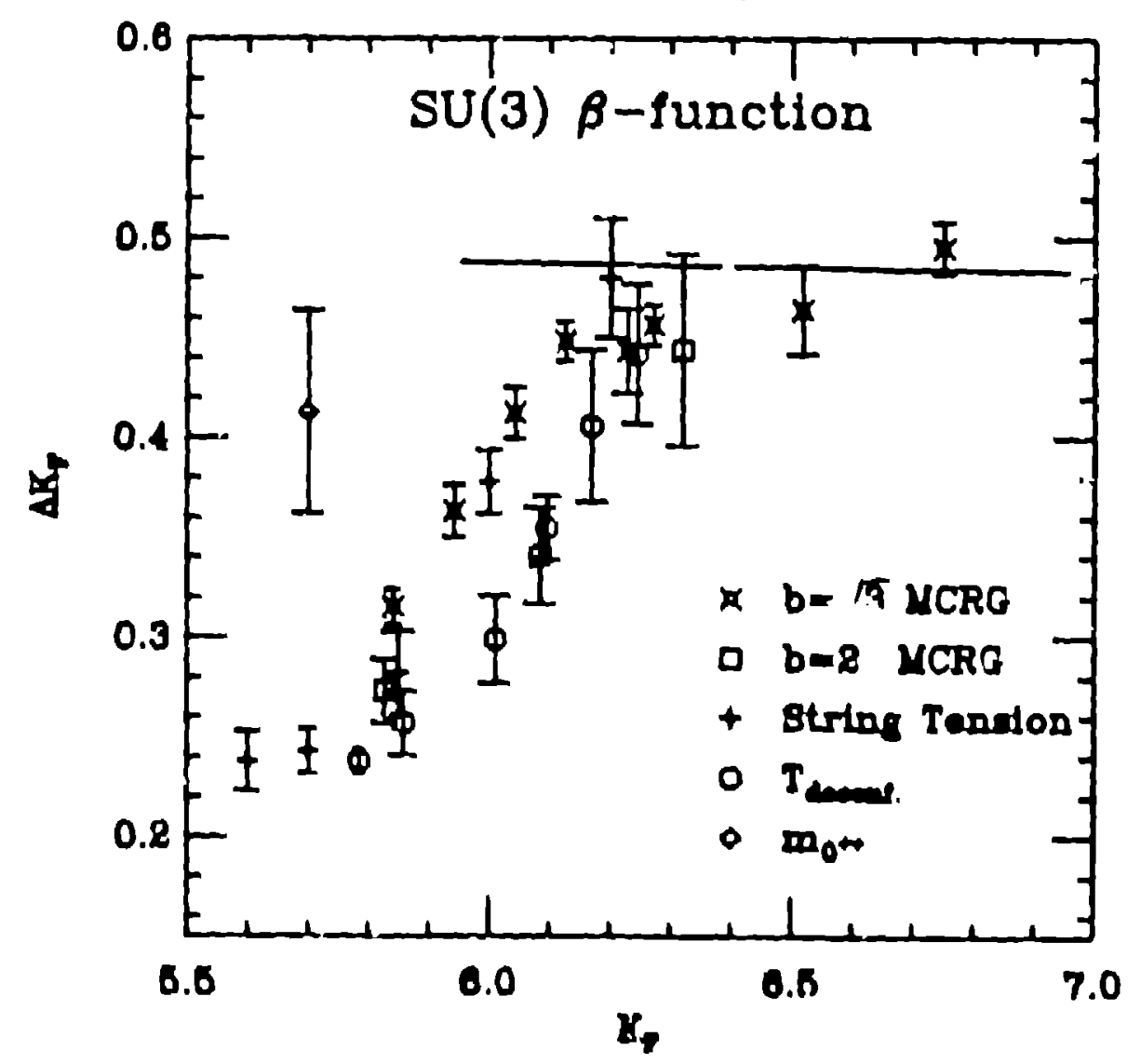

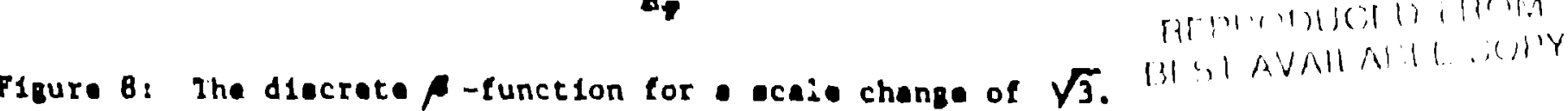


that are large for $g \sim 1$. Thus these calculations should be used as a guide and the goal should always be to attain constant mass-ratios.

To conclude this section; $M C R G$ calculations have not yielded any physical realts so far, but they have provided us with a definitive statement on the approach to the continuum limit. This is non-trivial. The present MC deteraination of $\sigma$ and the glueball masses need improvement before a definite statement of scaling can be made. The largest lattice calculation of $\sigma$ by de Forcrand 41 show deviations from aymptotic $x$ aling i.e. $\sqrt{\sigma}=92(79) \Delta_{L}$ at $\frac{\rho}{\beta^{3}}=6.0$ (6.3). Since these calculations have alresdy taxed the power of a Cray XMP-48, it leads us to the question whether improved actions can help. This is discussed next.

\section{B: DETERMINATION OF THE IMPROVED ACTION.}

The advantage of using an improved action in MC simulations in to reduce the effect of operators that lead to sraling viulations. In QCD this means that corrections to mass-ration determined from small lattices can be reduced. Second, we want to avoid regions near ningularities where continusm mass-ratios are violated. A known example is the end point of the phase structure in the fundamental-adjoint plane. There are, to the best of my knowledge, 11 methods in existence to calculate the renormalized couplings. All, except for those using perturbation theory (and therefore only valid near $g=0$ where scheme dependence is negligible), are based on $M C R G$. In fact, since the flxed point and the Renormalized trajecisry is a function of the $R G T$, an improved action is content-free unless the $R J T$ is specifled.

I shall briefly deacribe the methods, atate their advantuges and disadvantages and mention results obtained with them. The generic problem of systematic errors in the estimate of the couplings due to truncation in the number of couplings kept in the analyois will be referred to as "truncation errors". This is a seriou drawback because the errorn can be very large and there is no way of estimating them without a second long simulation. In order to consider this truncatecl ansetz to bo the best 'fit', a criterion to Judge the improvement has to ha established. This b discussed after a brir. deacription of the methors. To Ex the notation, the pure gauge SU(2) action is written as

$$
\begin{gathered}
S=K_{r} \sum \operatorname{Tr} U_{p}+K_{O P} \sum \operatorname{Tr} U_{O P}+K_{A} \sum\left\{\frac{1}{3}\left(\operatorname{Tr} U_{p}\right)^{2}-\frac{1}{3}\right\} \\
+K_{I} \sum\left\{2\left(\operatorname{Tr} U_{p}\right)^{s}-\operatorname{Tr}_{P}\right\}
\end{gathered}
$$

while the SU(3) action b

$$
\begin{aligned}
S=\operatorname{Re} \mid K_{r} \sum \operatorname{Tr} U_{\mathrm{r}} & +K_{\mathrm{Op}} \sum \operatorname{Tr} U_{\mathrm{Op}}+K_{0} \sum\left\{\frac{3}{2}\left(\operatorname{Tr} U_{\mathrm{p}}\right)^{2}-\frac{1}{2} \operatorname{Tr} U_{\mathrm{p}}\right\} \\
& \left.+K_{A} \sum\left\{\frac{9}{8}\left|\operatorname{Tr} U_{\mathrm{p}}\right|^{2}-\frac{1}{8}\right\}\right),
\end{aligned}
$$

Here the higher represontations have boun conntructed from $U_{p}$, all the traces are normalised to unity and the aume are over all altee and ponitive orientatione of the loops. 


\begin{tabular}{|c|c|c|}
\hline \multicolumn{3}{|c|}{$\Delta B$ for $S U(3) \quad\left(16^{4}\right.$ matched with $\left.8^{4}\right)$} \\
\hline $\boldsymbol{K}_{\boldsymbol{p}}$ & $b=2$ MCRG method & $b=2$ 1-loop Ratio method \\
\hline 6.0 & $0.35(2)$ & i).36(3) \\
\hline 0.3 & $0.43(3)$ & $0.45(3)$ \\
\hline 6.6 & $0.55(9)$ & \\
\hline
\end{tabular}

Table 2: The values of $\Delta \beta$ for a scale change of $b=2$. The renults are from Haseufratz a al ${ }^{24}$.

\begin{tabular}{|c|c|c|c|c|}
\hline $\begin{array}{c}\text { Initial } \\
\text { Action } \boldsymbol{K}_{F}\end{array}$ & $\boldsymbol{K}_{\boldsymbol{r}}$ & $\frac{K_{A}}{K_{F}}$ & $\frac{K_{2 / 2}}{K_{p}}$ & $\frac{K_{G p}}{K_{p}}$ \\
\hline $2.50(W)$ & $\begin{array}{l}2.571(05) \\
2.058(06)\end{array}$ & $\begin{array}{l}-0.193(01) \\
-0.186(06)\end{array}$ & $\begin{array}{l}0.043(01) \\
0.038(03)\end{array}$ & $\begin{array}{l}-0.0036(003) \\
-0.010(02)\end{array}$ \\
\hline $2.75(W)$ & $\begin{array}{l}3.16(1) \\
2.815(35)\end{array}$ & $\begin{array}{l}-0: 99(03) \\
-0.214(11)\end{array}$ & $\begin{array}{l}0.042(02) \\
0.044(06)\end{array}$ & $\begin{array}{c}-0.0208(015) \\
-0.019(04)\end{array}$ \\
\hline $3.00(w)$ & $\begin{array}{l}3.69(1) \\
3.469(46)\end{array}$ & $\begin{array}{l}-0.190(04) \\
-0.211(12)\end{array}$ & $\begin{array}{l}0.040(02) \\
0.039(04)\end{array}$ & $\begin{array}{l}-0.0314(007) \\
-0.032(03)\end{array}$ \\
\hline $3.25(W)$ & $\begin{array}{l}4.12(2) \\
4.003(37)\end{array}$ & $\begin{array}{l}-0.160(05) \\
-0.182(10)\end{array}$ & $\begin{array}{l}0.025(03) \\
0.032(06)\end{array}$ & $\begin{array}{l}-0.0374(004) \\
-0.040(03)\end{array}$ \\
\hline $3.50(w)$ & $\begin{array}{l}4.71(2) \\
4.396(67)\end{array}$ & $\begin{array}{l}-0.168(05) \\
-0.150(15)\end{array}$ & $\begin{array}{l}0.028(03) \\
0.007(06)\end{array}$ & $\begin{array}{l}-0.0402(004) \\
-0.049(02)\end{array}$ \\
\hline $4.35(\mathrm{MK})$ & $\begin{array}{l}3.42(1) \\
3.098(33)\end{array}$ & $\begin{array}{l}-0.211(02) \\
-0.235(12)\end{array}$ & $\begin{array}{l}0.044(01) \\
0.055(04)\end{array}$ & $\begin{array}{l}-0.0268(011) \\
-0.029(03)\end{array}$ \\
\hline
\end{tabular}

Table 3. Projaction of the renormalleed SU(2) action on to the $\left[\boldsymbol{K}_{\boldsymbol{F}}, \boldsymbol{K}_{\boldsymbol{A}}, \boldsymbol{K}_{\boldsymbol{N} / 2}, \boldsymbol{K}_{G}\right]$ ipace for meveral starting acticns. For each startud actlon, the fint row whowe the couplloge after one $b=\sqrt{ } 3 R S T$ on sta-tine lattloes of size $9^{4}$ calculated by the 2Lattloo mothod 2\%. The moond row shuwe the couplinges after Iwo RGT on etartIn lattloes of alze $18^{4}$ calculnted ung the microcanonlcal demon mothod os. The lant $20 t, K_{F}=4.35$, te on the MK trajoctory Eq. (5.6). 
5.1) Symarzlk Program ${ }^{45}$ : This is a perturbation theory method to remove all $O\left(a^{2}\right)$ corrections in physicai observables. At the tree level, at $1-$ loop $^{46}$ and in the leading $\log ^{47}$ anslysis, the $O\left(a^{2}\right)$ corrections are removed by including the 6 -link flanar loop with otrength

$$
\frac{K_{O_{P}}}{K_{\boldsymbol{F}}}=-0.05
$$

There have been some SU(3) calculations ${ }^{30}$ done with this action, but, they are inconclusive and no statement for an improvement in mass-ratios can be made.

6.2) Block Spln Renormalle ation Group (perturbation theory): The first work in this direction is by Wilson' who wrote down the ansatz

$$
\frac{K_{\text {op }}}{K_{r}}=-0.0576, \frac{K_{\text {ol }}}{K_{r}}=-0.0388 \text {, }
$$

where $K_{0 t}$ is the twisted 6-link coupling. No calculation of physical observables has been done with this action. The group of Iwasaki et al. ${ }^{40}$ have made a large independent effort in this direction of improvement. They find that near $g=0$ the action after $3 R G T$ can be approximated by including the 6 -link planar loop with strength

$$
\frac{K_{\text {OP }}}{K_{\mathrm{r}}}=-\frac{0.331}{3.648} .
$$

They show that for both the Wilson ensatz, Eq. (5.1), and frr this action instantons are stable on the lattlce. Cince this is not true of the simple plaquette action, they regard it as another criterion for improvement. They have recently calculated the string tension and the hadron masses in the quenched approximation using the improved gauge action of $E_{4} .(5.5)$ and the standard Wilson action for the quark propagator on $12^{2} \times 24$ lattice at an effective $\frac{8}{p^{2}} \sim 5.9$. Their results for mass ratios are impresoive. A comparison with an equivalent calculation on the Wilson nxis is limited because a number of parametery are different. The accuracy of their results warrents more attention.

8.3) Migdal-Kadanoff Rocuralon Tecbnlque: This calculation ${ }^{40}$ is limited to the plaquette in the tundamental and higher representations. The integration over links is done by "spanding the action in ternu of the characters and then using the recursion formula. In the Improved action, the effect of the singularity in the fundamenta! adjoint plane is reduced but the leading irrelevant coupling $K_{\text {op }}$ is not included. For $\mathrm{SU}(2)^{40}$, the convergence in the character expansion was good, the recuraton was atable on keeping 20 characters. The Improved action is dominated by the opin 1 and $3 / 2$ representations, and the K-M improved trajoctory was approximaled hy

$$
\frac{Y_{A}}{K_{\boldsymbol{r}}}=-0.24 \text {. }
$$

It was later ahown by Bitar et al. ${ }^{\mathrm{B}}{ }^{\circ}$ that tile heat Kernal action works very nell in the rexuraion scheme and in iact $b$ the solution In the perturbative lirnit. For u SU(2) calculation of the $\beta$-function along the $\mathrm{K}-\mathrm{M}$ improied trajertory $K_{A}=-0.21 K_{\boldsymbol{F}}$, and for an analyais of the improved actlon nef. 22b.

6.3b) Phenon:enological (Llnes Of Conotant String Tenu: on): The conthuum limit in taken aiong directlons perpendicular to the lines of constant string 
tension in the negative fundamental- adjoint plene. Rebbi ${ }^{32}$ et al. have measured the $q \Phi$ fotential, while Samuel ${ }^{83}$ has spearheaded a calculation with walar quarks. The effective coupling for comparison on the Wilson axis is defined by usirg the large $N$

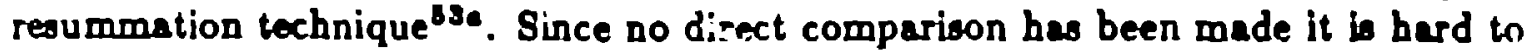
state if better maso ration are obtained.

5.4) Swendeen'e method ${ }^{84}$ uolng the Callen representation: The block expectations values of Wileon loope are calculated in two ways. Firat as eimple averages over block configurations, and recond uning the Callen representationss with a guess for the block couplings. From these two eatimaten, the block couplingo are determined iteratively. The method is fast and easy to implement. It does have undetermined truncation errors. Lang ${ }^{80}$ has used this method to show that the quartic coupling $\lambda \phi^{4}$ in the eelf-interacting scalar feld theory renormalizes to zero. Recently Burkitt ${ }^{35}$ has used it to map the flow of the action under the $b=2 R G T$ (sertion 2.2) for the $U^{(1)}$ model. From a difference in the flows he can eatimate the transition point on the Wileon axas. It would be instructive to extend the III(1) andyois to ty couplinf. values along the phase transition line and check if there is a qualitative change at the $T C P$.

6.5) Callaway-Petronzlo- WHson ${ }^{57, k 8}$ method of Axed tlock aplas: This method is uacful for dincrete spin systems like the lsing model and models in the same univeronlity class. A $M C R G$ calculation is modilied by fixing all the block spins except one such that only a controllable few block interactiors are non-zero. The system in aimulaled with the RGT used as an addit'onal weight in the Metropolis algorithm. The ratio of probabiiity of this unfixed spin being up to it being down is equal to a determined function of a certain number (depending on how many block interactions are nun-zero) of block couplings. By using different confgurations of fxed block spins a system of lis:ear equations is set up from which the block couplings are determined. The drawback of this method, even for the lving model, is that it is hard to set up the black opins so that only a few $(\approx 10)$ block interactions are nonzero. Wilson showed that this can be done if one uses the lattice gas repreasentation i.e. 0 or 1 for apin values. The couplinge in the \pm 1 representation are then given by an expansion in the lattice gas couplings. This expansion has been showr to converge rapidly for tlie $d=2$ lsing model. Thr eecond improvement due to Wilson is that instead of $\triangle \mathrm{MC}$ delermination of the ratio of probab'iiles, the exact result can be obtained in the tranofe" matrix formaliom. The drawback here is that a Vax 11/780 type wechine can hendle only up t $v$ 1s spins. However ito non-otatistical nature makes it uneful a a test.

6.6) Character Expanalon mathod of Bltar ${ }^{80}$ : I will deacribe this metliod with a restriction to simple plaquelte actions. The character expansion for the uction is $\quad S=\sum_{p} \sum_{r} K_{r} \chi_{r}\left(U_{p}\right)$ where $x_{r}$ is the character in the $r^{\text {th }}$ representacion and $K r$ is the corresponding culupling. Similarly the Bolezmenn factor $F_{p}$ for earh plaquette $p$ can be expanded in a clasacter expansion $F_{p}=\sum_{r} d_{r} f_{r} x_{r}\left(U_{p}\right)$ w'iere $d_{r}$ is the dimension and $f_{r}$ the coefllcient for $r^{\text {th }}$ ropresentation. The couplings $Y_{r}$ are given by

$$
K_{r}=\int d\left(U_{p}\right) \ln F_{p}\left(l_{p}^{\prime}\right) x_{r}\left(L_{p}^{\prime}\right)
$$

The cruclal atep is that the ratio $\frac{d}{\}_{1}}$ can be calculated as a ratio of expectation val- 
ues mer block conflgurations. From this the Boltzmann factor $F_{p}$ and consequently $K_{\mathrm{r}}$ can be determined. The metind is sensitive to the convergence of the character expansion i.e. the r.unber of terms in, sicsded to determine $F_{p}$ accurately. After this there are no trunestion errors in determining $K_{r}$. The method grows in complexity if lerger loops are to be included in the analysis. The frst results ${ }^{B 0}$ for the simple plequette action in $\mathrm{SU}(2)$ are encouraging.

6.7) The Schwinger-Dyson Equation method of Falconi et al..$^{\circ 0}$ : In this method the lattice Schwinger-Dyson equations (equations of motion for expectation valuen of , point functions) are used to write down a set of inhomogeneous linear equations for the couplings. The coefficients and the inhomogeneous term are given in terms of expectation values of n-point functions. In deriving these equations the action has to be truncated to the aubspace of couplings to be determined. Thus the method has truncation errors. Preliminary results for the $O^{\prime}(3)$ non-linear $\sigma$-model in $d=2$ are encouraging.

B.R) 2-Lattice $M C R G$ method 01,0 : The calculation steps are the same as Wilson's 2-Lattice method to determine the $\beta$-function. The method consists of expanding the block expectation value, (with unknown couplings) about those from a simulation with known couplings. Keeping jusi the linear torm is the expansion gives the difference between the two sets of couplings. The main advantage is that this comes free with the calculation of the $\beta$-function. The method has a atatistical drawback that it requires two different simulatione so there is no possibility of cancellation of statistiral errors. Also, far from the $R T$, only the first renormalized couplings can be determined axcurately. Thers exist extensive calculations for both the SU(2) and the SU(3) models using tine $\sqrt{3} R G T$. The estimate for the improved action in a 4-parameter opace for $S U(2)$ is ${ }^{22}$

$$
\frac{K_{O P}}{K_{F}}=-0.06, \frac{K_{A}}{K_{F}}=-0.1 \theta, \frac{K_{F}}{K_{F}}=0.03
$$

and for SU(3) is ${ }^{37}$

$$
\frac{K_{O_{P}}}{K_{F}}=-0.04, \frac{K_{B}}{K_{F}}=-0.12, \frac{K_{0}}{K_{F}}=-0.12 .
$$

The truncation errory are known to be large and the reliablicy of the results in being tested by using the ootimrted inproved action in the pdate and repeating the calculation of the $\beta$-function and the improved $n$.tion. Also the hadron spectrum is being calculated to test if better mass-rotios are ubtained. A detailed comparason of the results for the renormalized action is made with the microcanoniral method discussed next.

6.9) T.ilcrocanonleal (Creute's Demon) Method ${ }^{02}$ : Thlo method lo very efficient if from a previoue $M C R G$ calculation expectation values of $n$ block Wiloon loops it each of the $I$ block levels are determined. To determine the correnponding couplinge at the $1^{\text {th }}$ level, = microcanonical amulation ta then done (on a same nize lattlce $a$ on which the block expertation values were calculated) with the corresponding $n$ energies bxed and with one demon per Inturaction. The desiacd $n$ couplings are then determlited from the distribution of demon energies. P. Stolorg ${ }^{03}$ 
-t Caltech used the block expectations values obtained after two applications of the $\sqrt{3} R G T$ for SU(2). From these he obraived the cecond, $(l=2)$, renormalized action in a truncated ccupling constant brare (four couplings of Eq(5.1)). The results are shown in Table 3 and comparad with the first renormalized couplings obtained from the 2-Lattice $M C R G$ method described above. The results show a rapid convergence of the action to the $R T$ cunsistent with the estimutes given in Eqc (5.8). This is evidence that the $\sqrt{3} R G T$ transformation has good convergence propeities after two steps. In this calcuintion it was easy to thermalize the four energies. The simulation is faster thnn the 2-Lultice michod and has better statistical properties. Also the block couplings at all levels can be determined once the block expectation values are known. The truncation ercors ar he same as in the 2-Lattice method.

b.10) Block Dlagonalization method of Mutter and Schlllng ${ }^{04}$ : This is at present the only ar:ethod the: attempts to improve botb the gauge =id the fermion action. The main itea is thet quark proprgators are calculated on blocked gauge configurations using a blocked fermion action. The blocked fermion action is calculated as follow: Let the starting action be the Wilson action

$$
\boldsymbol{\Psi} \boldsymbol{\Psi} \boldsymbol{\Psi},
$$

wherc $M$ is the interaction matrix. The lattice is now divided inio blocks which for the $\sqrt{3} R G T$ contain 9 sites each. The site action is then cast into a block artion

$$
\text { ETE }
$$

where $\Xi$ is a 9 component Dirac fermion feld and $I^{\prime}$ is the interaction matrix set up to reproduce Eq. (5.10). The mass term part of $\Gamma, \Gamma_{m}$, is now diagonalized to provide the non-interaction fermion basis vectors. For the $\sqrt{3} R G T$, tho 9 eigenvalues of $\Gamma_{m}$ are 0 and 8 degenerat $A$ ones with value $\frac{?}{a}$. The light mode alone is kept on the blocked lattice. The interartion between the light and heavy modes is calculated in perturbation theory and inese terms a-a added to the Wilson action to give the improved fermion coupling matrix. It !s to be noted that hiis fermion diagonalization is or.ly approximate. Thur lattice masses will not a priori change by the acale factor $b$ between the original and the blocked lattice. It is therefure necessary to flust check huw good the transformation in in preserving mass-ration of the unblocked aystem. The results on 2 twice blocked set of centigurations using $b=2$ ere encouraging ${ }^{\circ}$. Results of a test of preservation of mass ratios under blocking shoulc be available soon for both the $b=2$ ard $l=: \sqrt{3} R G T$. At this point it is worth mentioning that the following advantages were observed in the diagonalizatiun proress for the $\sqrt{3} R G T$ in comparion to $b=2$.

(a) The seperation between the light modes $m \sim 0$ and the heav' modes is bette i.e. versus $\frac{2}{a}$, on the perturbativa corrections we more reliable.

(b) Rotational invariance in not broken as b th the $b=2$ tranaforniation.

(c) No ilosed gauge loops which manlfest themselves as adilitional contact terms in the fermion operators arloc. This Implles that the value of the Wilson parameter $r$ does not get nodlfied and $\boldsymbol{\kappa}_{\mathrm{u}}$ would remain the uarric on the blocked lattice for the Wileon fermiona if the exact ferralon couplline matrix was used in the calculations.

(d) The blocking of gauge links th the same ss defined in soction 2.3 . 
Discusslon: There are some features of the improved action that seem common to the various analysis done. The details will certainly depend on the specific RGT.

(a) The leading irrelevant operator is dominated by $K_{\theta p}$, the 6-link planar Wilson lcop. Thus a $R G T$ that kills it is an improvement.

(b) From the $\sqrt{3} R G T$ inalysis, one gets an estimate of $\frac{K_{A}}{K_{r}} \sim \frac{K_{R}}{K_{r}} \sim-0.12$. Thus near $\frac{a}{0^{2}}=6$, the phase structure in the $\left\{K_{F}, K_{A}\right\}$ plane is avoided. This is necessary because in the vicinity of the end point of the phase structure universality is violated.

(c) The $R T$ for the $b=\sqrt{3} R G T$,how's significant deviations trom linearity in the region accessible to Monie Carlo. The ratios given in Eqs. (5.8) and (5.8) are an estimate of the arymptotic behavior.

(d) The $R T$ out of the fixed point is local i.e. dominated by small loops. The Wilson axis is tangent to the strong coupling $R T$ at the trivial fixed point at $K_{a}=0$. The change from the weak coupling $R T$ to flow close to the Wilson axis takes plave in the region where current Montc Carlo calculations have been done i.e. between 5.7 and 6.5. This feature needs to be investigated since curient massratios show a behavior that is in between strong coupling and the expected continuum one.

IL is otill necessary to evaluate whether constant mass-ration in the quenched appraximation are obtained earlier with an improved action. The results have to justify the factor of $\sim 5$ by which the gauge update slows down when the above four couplings are used. The key lies in improving the fermion sector. For dynamical quark, the gauge update is a smail fraction of the update time. So, an investment in imroving the action is justified.

\section{6: MMPROVED MONTE C ARLO RENORMALIZATION GROUP}

I shall review the Gupta-Cordery Monte Carlo Renormalization Group method (IMCRG) in some detail. In this method the Renormalized Hamiltonian and the Linearized Transformation Matrix, LTM, are determined without any truncation errors. There are no long time correlations even on the critical ourface and the block $n$-point correlation functions like $\left\langle S_{o}^{1} S_{\beta}^{1}\right\rangle-\left\langle S_{a}^{1}\right\rangle\left\langle S_{\beta}^{1}\right\rangle$ are calculable numbers. Also, the method allows a careful orror analysis in the determination of the renormalized couplings and in the LTM.

In the IMCRG method the configurations $\{s\}$ are generated with the weight

$$
P\left(s^{1}, s\right) e^{-H(a)+H^{\prime}\left(a^{1}\right)}
$$

where $H^{0}$ is a guess for $H^{1}$. Note that both the site and block spins are used in the update of the site spine. In analogue to Eq. (1.2), the distribution of the block spins is given by

$$
e^{-H^{1}\left(0^{1}\right)+H^{\prime}\left(0^{1}\right)}=\sum P\left(0^{1}, 0\right) e^{-H(0)+H^{0}\left(0^{1}\right)} \text {. }
$$

If $H^{\theta}=H^{1}$, then the block spins are completely uncorrelated and the calculation of the n-point functions on the block lattice is trivial.

$$
\left\langle S_{a}^{1}\right\rangle=0 \quad\left\langle S_{a}^{1} S_{\beta}^{1}\right\rangle=n_{a} \delta_{a \rho} \quad \ldots
$$


where for the Ising mode! (and most other models) the integer $n_{a}$ in simply a product of the number of sites times the multiplicity of interaction type $S_{a}$. When $B^{\theta} \neq B^{1}$, then to first order

$$
\left\langle S_{\alpha}^{1}\right\rangle=\left\langle S_{\alpha}^{1} S_{p}^{1}\right\rangle_{H \cdot-H^{1}}\left(K^{1}-K^{0}\right)_{\rho}
$$

and using Eq. (6.3), the renormalized couplings $\left\{K_{a}^{1}\right\}$ are determined with no truncation errors as

$$
K_{a}^{1}=K_{a}^{0}+\frac{\left\langle S_{a}^{1}\right\rangle}{n_{a}} .
$$

This procedure can be iterated - use ${H^{n-1}}^{n}$ the opin $B$ in Eq. (6.1) to find $H^{n}$. If the irralevant eigenvalues are small, then after two or three repetitions of the $R G T$, the sequence $K^{n}$ converges to the fixed point Hamiltonian $H^{*}$ which is assumed to be short ranged. For the $d=2$ Ising model, the method has been shown to be extremely stable ${ }^{07}$. The only limitations of this method are the linearity approximation, Eq. (3.4), (this is trivially handled by iterating $H^{0}$ ) and the use of a truncated $B^{n-1}$ for the spin Hamitonian in the update to find $H^{n}$. The second limitation can be overcome and the solution in straightforward: In Eq. (6.1) use $H^{0}$ as the guess for $H^{n}$. The updatc now involves the original spins and all block spins up to the $n^{\text {th }}$ level in tie Boltzmann weight

$$
P\left(a^{n}, a^{n-1}\right) \ldots \ldots P\left(a^{1}, s\right) e^{-H(o)+H^{0}\left(a^{n}\right)} .
$$

The four Eqs. (6.2-6.5) we unchanged except that the level superscipt is replaced by $n$, i.e. the $n^{\text {th }}$ level block-block correlation matrix is diagonal and given by Iq. (6.3). With this modificaiion, the $H^{n}$ is calculated directly. The limitation on $n$ is the size of the starting lattice. Such a check is necessary because errors in long range couplings due to finite statistica and the effects of a truncation in the spin $H^{n-1}$ get magnified and the system rapidly flows away from the fixed point.

The calculation of the LTM proceeds exactly in the standns $M C R G$ i.e. Eqg. (1.4) to (1.6). However, in the limit $H^{0}=H^{1}$, the block-binck correlation matrix is diagonal and given by Eq. (6.3). Thus it has no truncation errors, can be inverted with impunity and the final LTM elements are also free of all truncation errors. The only error is in finding the eigenvalues from a truncated matrix. These errors can be estimated and the results improved as explained below.

$I M C R G$ is therefore more complicated than $M C R G$ and requires a simultaneous calculation of a many term $H(s)$ and $H^{g}\left(s^{1}\right)$ at update. However, the system does not have critical slowing down. Secondly, the correlation length $\xi$ can always be made of $O(1), w$ finite size effects are dominated by the range of interactions, which by assumption of a short range $H^{*}$ fall off exponentially. Thus, critical phenomenon can be atudied on small lattices and with no hidden aweep to sweep corrolations that invalidate the atatistical accuracy of the results.

\section{1: Tiuncatlon Errors In The LTM}

Consider the matrix a quation for $T$ in block form

$$
\left(\begin{array}{ll}
D_{11} & D_{12} \\
D_{21} & D_{22}
\end{array}\right)\left(\begin{array}{ll}
\Gamma_{11} & T_{12} \\
T_{21} & T_{22}
\end{array}\right)=\left(\begin{array}{ll}
U_{11} & U_{12} \\
U_{21} & U_{22}
\end{array}\right)
$$


where $D_{11}$ and $U_{11}$ are the 2 derivative matrices calculated in some truncated space of operators that are considered dominent. The elements of the sub-matrix $T_{11}$ will have no truncation errors provided $w:$ can calculate

$$
T_{11}=D_{11}^{-1}\left\{U_{11}-D_{12} T_{21}\right\} .
$$

In the IMCRG method the matrix $D$ is diagonal and known, so $D_{12}$ is 0 . Thus elem 'nts of $T_{11}$ determined from $U_{11}$ have no truncation errors. The crrors in the eigerivalues and eigenvectors arise soicly from diagonulizing $T_{11}$ rather than the ${ }^{\prime}$ Il matrix $T$. Calculations in the $d=2$ Iaing model have shown that these errors are large, i.e. of order $10 \%$, if all operators of a given rauge are not included. An open problem right now is a robust criterion for classifying operators into sets such that including successive sets decreases the truncation errur geometrically by a large factor.

The errors arising from using a sub-matrix $T_{11}$ can be reduced sigr ificantly by diagonalizing

$$
T_{11}+T_{11}^{-1} T_{19} T_{21}=D_{11}^{-1} U_{11}+\left\{-D_{11}^{-1} D_{12}+T_{11}^{-1} T_{12}\right\} T_{21}
$$

as shown by Shankar, Gupta and Murthyce. The correction term $T_{11}^{-1} T_{12} T_{21}$ is the $2^{\text {nd }}$ order perturbation res:it valid for all eigenvalues that are large cumpared to those of $T_{22}$. This correction matrix car be colculated in IMCRG from $\left(T^{2}\right)_{11}-$ $\left(T_{11}\right)^{2}$. I am here o'erlooking the errors due to the $R G$ flow, because of which $T^{2}$ is evaluated a a different point than $T$. Another mspect of these errors is their behavio: as a function of how close to $H^{*}$ the calculation is done. For the $d=2$ Ising model we $\mathrm{e}^{06,60}$ find that the truncation errors in the relevant eigenvalues are large. Adding more operators does not monotonically decrease the error. The fluctuations can be as large as $2 \%$ even after the 20 largest operators are included in $T_{11}$.

In st undard $M C R G$, the calculations with $T_{11}=D_{11}^{-1} U_{11}$ have shown good convergence once lew operators, $O(5-10)$, are included in $T_{11}$. The reason for this is an approximate cancellation of a term ignored and the correction term. Using Eq. (6.7), ignoring term. with $T_{22}$ and approximating $T_{11}=D_{11}^{-1} U_{11}$ we get

$$
-D_{11}^{-1} D_{12}+T_{11}^{-1} T_{12} \sim-D_{11}^{-1} D_{12}+U_{11}^{-1} U_{12} \text {. }
$$

Further, these derivative matrices are roughly proportional, i.e. $U \sim \lambda_{t} D$ and the corrections fall off as the ratio of non-leacing eigenvalues to the leading one $\lambda_{1}$. This follows from the arguments of section 1.1 and can be checked by expanding oparetors in term of eigenoperators. Thus Swendsen ${ }^{7}$ by calculating just $D_{11}^{-1} U_{11}$ and ignoring all truncation problems was effectively incorporating a large part of the perturbative correction piece. This explains his success. Shankar ${ }^{70}$ has found a correction term to further decrease the truncation effects in $M^{\prime} ; R G$. However, given the assumptions, the flow under $a R G$ and the success of the procedure as it exista, an improvement may be liurd to evaluate.

Thus, at present the best way to get accurate rasults is to use IMCRG to calculate the Renormalized couplings and Swerdsen's $M C R G$ method to calculate the elgenvalues. The topics that need more work are the accuracy of perturbative improvement In $I M C R G$, the clausification of interactions Into complete sets and a quantitative underetanding of the tuning of the RGT. 
Let me alno summarize some of the other results obtrined from the study of the $d=2$ Ising model.

[1] The LTM has elements that grow along row: and fall along columns ${ }^{\text {ob }}$, therefore it can be arranged to look like

$$
\left(\begin{array}{ll}
A & B \\
C & D
\end{array}\right)
$$

with $A$ the minimal truncated $n \times n$ block matrix that shou!d be calc slated. The case $\varepsilon=0$ is aimple; there are no truncation errors in either method and diagonalizing $A$ gives the $n$ largest eigenvalues. Otherwise for $I M \cong R G$ the truncation errir depends on the dot product of terms in $c$ and $B$. The requirement of absolute convergence in the dot produci only guaroniee that this product is finite but it may be arbitrarily large i.e. $O(1)$. Therefore fo, each model, a careful study of the signs and magnitude of the elements in $c$ as a function of the RGT becomes necessary. This is being tone at Cornello".

[2] The leading left eigenvector is normal to the critical surface ${ }^{6 \theta}$. Its elements give an estimate of the growth in the elements along the rows of the LTM.

[3] Using $H^{0}$ as the known nearest-neighbor sritical point $K_{n n}^{c}=0.44068$, the $I M C R G$ results ${ }^{07}$ for $H^{1}$ are independent (within statistical accuracy) of finite size effects for lattice sizes $16,32,64$ and 128 .

[4] The results for $B^{n}$ converged provided the couplings in $H^{0}$ were correct to $O\left(10^{-3}\right)$. This initial accuracy can be achieved ${ }^{67}$ with a few thousand sweeps on a $128^{2}$ lattice.

15] The statietical erron in IMCRG can be evaluated very reliably ${ }^{07}$. Detailed binning analysis showed that each sweep is approximately independent and ar. accuracy of $10^{-8}$ is obtained in all couplings with $\sim 2 \cdot 10^{\circ}$ sweeps on a $64^{2}$ lattice. This could be achieved with $3000 \mathrm{Vax} 11 / 780$ hours.

To conclude, I believe that IMCRG provicies a complete framework to analyze the critical behavior of spin and gauge models. With the increased availability of supercomputer time we shall have very accurate and reliable results.

\section{7: EFFECTIVE FIELD THEOPIES}

The point of effective field theories is that physical phenomena at some gir in length scale can be described by some effective/compositu degrees of freedon. The couplings between these variables are determined by the underlying microscopic theory. Thus we would like to know these effective degrees of freedom and the couplirgs. So far the discuseion of $M C R G$ has focused on the change of acale without a change of varinbles. To make full use of its power, a transformation of variables at the appropriate acale nhould be added i.e. In addition to a $R G T$ that just cverages over degrees of freedom, consider a change from the microscopic theory to an effective theory with naw ra-iables at some give length acale. These variables can be composite (as lo the case in goling from QCD to a theory where the degrees of freedom are hadrorin) cr represent a freezing as in SU(2) at high temperatures where the interaction between ti: Wilson lines lo describer by an effective $d=3$ Ining movel. Here one tranafo' ma írom link variablen to Wilann linat to Inina enina 
Once the effective theory has been constructed, it is important to know the universality clase to which it belongs. This would provide a detailed knowledge of the critical/long distance behavior. Little work has been done in actually exploring univeradity clases by mapping flows that incorporatis a change of variables.

The way to do this in standard $M C$ is to define the composite degrees of freedom and their n-point functions in terms of the microscopic variables. From the expectation values of these n-point correlation functions calculated as simple averages, the corresponding couplinge can then be determined by a Microcannonical simulation as deacribed in eection 5.9. One ouch calculation is by Ogilvie and Gockech ${ }^{70}$ in which they determine the nesrest neighbor couplings between the Wilson lines in SU(2).

In $M C R G$, the transformation from the microscopic degrees of freedom to the composite variables is made on the original lattice (same as in $M C$ ). The $R G T$ is defined on the componite variables and the critical exponents of the effective theory are calculated from the LTM. The couplings can be determined by one or more of the methods of cection 5. This process also maps the universality class. Similarly, $I M C R G$ is be used provided $H^{0}$ in a guessed hamiltonian for the effective theory. This aubject to being actively puraued in collaboration with A. Patel, C. Umrigar and K. G. Wibon and we hope it will blowom.

\section{ACKNOWLEDGEMENTS}

I would like to thank my collaborators R. Cordery, G. Guralnik, G. Kilcup, G. Murthy, M. Novotny, R. Shanker, S. Sharpe, C. Umrigar, K. Wilson and expecially A. Patel for tho wor's presented here and for many long and fruitful discuesions. It is a pleasure to acknowledge the hoopitality of K. H. Mutter and navor the experience of warching him attend to details necessary to make this an exciting conference. 


\section{RTFERESTCNS}

[1] K. G. Wilson, in Recent Developments in Gauge Theories, Cargese (1070), eds. G. t' Hooft, et al. (Plenum, New York, 1980).

(2) R. H. Swendsen, Phys. Rev. Lett. 12, (1979) 85s

[3] S. H. Shenker and J. Tobochnik, Phva. Rev. B22 (1980) 4162.

(4) S. K. Ma, Phys. Rev. Lett. 87, (1976) 461.

(5) L. P. Kadanoff, Rev. Mod. Phys. 40, (1077) 267.

[6] K. G. Wileon, in Pragress in Gauge Ficld Theories, edited by G. 't Hooft et al., (Plenum, Now York 1984).

(7) R. B. Swendsen, in Real Space Renormalizition, Topice in Current Phyoics, Vol 30, edited by Th. W. Burkhardt and J. M. J. van Leeuwen (Springer, Berlin, 1082) Pr. 67.

[8] J. M. Drouffe and C. Itzykson, Phys. Reports 38 (1978) 133.

J. B. Kogut, Rey. Mnd. Phys. 61 (1979) 659 and 65 (1983) 775.

M. Creutz, L. Jacobs and C. Rebbi, Phys. Rep. 0i, (1983) 201.

M. Creutz, Quarks, Gluons and Lattices, Cembridge Univ. Press (1984).

J. M. Drouffe and J. B. Zuber, Phys. Reports 102 (1983) 1.

[y] K. Binder, in Monte Carlo Methade in Statiotical Physics, edited by K. Binder (Springer, Berlin,1979) Vol 7 , and in Applications of Monte Cario Methods in Statistical Physice, (Springer Verlag, Heidelberg, 1983).

(10) I hive taken the liberty to use as synonymous t'se terms action and Hamiltonian since the meaning is clear from the context.

(11) K. G. Wilson and J. Kogut, Phys. Rep. 12C, (1974) 70.

12] P. Jeuty and G. Toulouse, Introduction to the Renormalisation Group and Critical Phenomenon, (John Wiley \& Sons, New York :978).

[13] D. Anut, Field Theory, the Renormalization Group and Critical Pheromenon, (World Scientille, 1084).

[14] N. Metropolis, A. W. Rosenbluth, M. N. Rosenbluth, A. H. Teller and E. Teller, J. Cher. Phys. 21 (1053) 1087.

(15) M. Creuiz, Phys. Rev. D 21 (1980) 2308.

(16) D. Callaway and A. Rehmen, Phys Rev. Loit. 10 (1982) 613.

M. Creutz, Phy. Rov. Lett. 60 (1983) 1411.

J. Polonyl and H. W. Wyld, Phys. Rov. Lett. 61 (1983) 2257.

(17) G. Parbi and Wu Yongohi, Scl. Sin. 24 (1981) 483.

[18] G. U. Batrounl, G. R. Kats, A. B. Koinfeld, G. P. Lapage, B. Svetiteky, and K. G. Wilson, Cornell Preprint CLNS-85(65), May 1985.

(19) A. Brandt, In Multigrid Methode, Lecture Notew in Math 060, (Springer Verlag 1982) and refurencee theroin.

(20) The idea was Ant discused by G. Parbl in Progress in Gauge Field Theories, edited by G. 't Hooft, et al., (Plenum, New York, 1084).

(21) R. Swendsen, Phys. Rov. Lett. 47 () J81) 1773.

[22] R. Cordery, R. Gupte and M. A. Novotny, Phys. Lett. B 128 (1983) 428.

R. Gu ite and A. Patel, Nucl. Phyn. B 281 (J085) 789.

[23] D. Caliaway and R. Patronalo, Phyo. Lett. B (1085) .

This tranaformation wa abo known to R. Gupta, B. Svetitaky and K. G. Wilson but not puraued in favor of the firat three. 
[24] K. C. Bowler, A. Hasenfratz, P. Hasenfratz, U. Heller, F. Karsch, R. D. Kenway, I. Montvay, G. S. Pawley, and D. J. Wallace, Nucl. Phys. B257 (1985) [FS14] 155 , and

A. Hasenfratz, P. Hasenfratz, U. Heller, and F. Karsch, Phys. Lett. 140B (1984) 76.

[25] A more careful statement is made by M. E. Fischer and M. Randerir, Cornell Note (1985).

(26) R. H. Swendsen, Phys. Rev. Lett. 62 (1984) 2321.

(27) G. Bhanot, Nucl. Phys. B205 (1982) 168.

[28) H. G. Evertz, J. Jersák, T. Neuhaus and P. M. Zerwas, Nucl. Phys. B251 (1985) 279.

[29] The presence this phase transition was shown analytically by $\mathrm{A}$. Guth, Phys. Rov. D21 (1980) 2291; and by J. Frolich and T. Spencer, Commun. Math. Phys. 83 (1982) 111.

[30] T. Banks, R. Mayerson and J. Kogut, Nucl. Phys. B129 (1977) 493.

[31] T. A. DeGrand and D. Toussaint, Phys. Rev. D22 (1980) 2478.

[^2] R. Gupta, M. A. Novitny and R. Cordery, Northeastern Preprint 2654 (1984). A condensed version to appear in Phys. Lett. $\mathrm{B}$

[33] J. Barber, Phyn. Lett. B 147 (1984) 330.

(31) V. Groach, K. Jansen, J. Jérsak, C. B. Lang, T. Neuhaus and C. Rebbi, CERN preprint $4237 / 85$.

(35] A. N. Burkitt, Liverpool preprint LTH 138 October (1n85).

(36] P. du Furcrand and C. Roiesnel, Phys. Lett. $137 B$ (1984) 213, and 143B (1984) 453.

P. de Forcrand, Ecole Polytachn'que preprint A615.0784 (July 1984).

(37) R. Gupta, G. Guralnik, A. Patel, T. Warnock and C. Zemach, Phys. Rev. Lett. 63 (1984) 1721.

R. Gupta, G. Guralnik, A. Pate!, T. Warnock and C. Zemach, Phys. Lett. $161 \mathrm{~B}$ (1085) 352.

(3E, M. Creutz, Phys. Rev. D23 (1081) 1815.

[39] F. Gutbrod, DESY preprint 85-092 (1988).

[40] D. Petcher, Private Communication.

141 P. de Forcrand, G. Schlerholz, H. Schneider and M. Tuper, Phya. Iett. 143B (1985) 107.

[42] S. W. Otto and J. Stack, Phys. Rov. Lott. 82 (1981) 2328.

D. Barkal, K. J. M. Moriarty and C. Rebbi, Phys. Kev. D30 (1984) 1293.

[43] S. Gottlieb, A. D. Kennedy, J. Kutl, B. Meyer, B. J. Pendleton, R. Sugar and

D. Toussaint, Phya. Rev. Lett. 88 (1085) 1058.

[11 P. de Forcrend, in the proceedling of this conference.

(45) K. Symenalk, in Procedinge of the Trieste workshop on non perturbative field theory and QCD (dec. 1088), World Sclentlifc (1983) 61.

[46] P. Welos and R. Wohlert, Nucl. Phys. B230 (1281) 197.

(17) G. Curcl, F. Menotti and G. P. Paffutl, Phya. Lott. 130D (1053) 205.

(18) Y. Iweakl, preprint: UTHEP-118 (1083)

8. Itr $h, Y$. Iweenti and T. Yoohlo, preprint UTHEP-194 and UTHEP-146.

(49) K. M. Ditar, 8. Gottllab and C. Zachow, Phyo. Rov D20 (1982) 2853.

(50) K. Bltar, D. Duke and M. Jadid, Phy. Rev. D81 (1086) 14\%.

[51] D. Barka, K. J. M. Moriarty and C. Rebbl, Phyı. Rev. D30 (1984) 220 1 
[52] O. Martin, K. Moriarty and S. Samuel, Nucl. Phys. B261 (1985) 433.

[53) B. Grossmann and S. Samuel, Phys. Lett. 120 B (1983) 383.

A. Gonealeo Arroyo and C. P. Korthals Altes, Nucl. Phys. B205 [FS5] (1982) 46.

R. K. Ellis and G. Martinelli, Nucl. Phys. B135 [FS11] (1984) 93.

[54] R. H. Swendsen, Phys. Rev. Lett. 62 (1984) 1165.

[55] H. B. Callen, Phys. Lett. AB (1861) 161.

[56] C. Lang in The Proceedings of the Tallahassee Conference on Advances in Lattice Gauge Theory, (World Scientific, 1985).

[57] D. Callaway and R. Petronzlo, Phys. Lett. 130B (1984) 189.

[58] K. G. Wilson and C. Umrigar, unpublished.

[59] K. Bitar, FSU preprint SCRI-85-7 (1985).

[60] M. Fulcioni, G. Martinelli, M. L. Paciello, G. Parisi, B. Taglienti, Nucl. Phys. B265 (1086) [FS15) 187.

(61) R. Gupta and A. Patel, Phys. Rev. Lett. 83 (1984) 531 and R. Gupta ard A. Patel in Proceedings of the Argonne Conference on Gauge Theory On a Lattice, (1984).

[62] M. Creutz, A. Gocksch, M. Ogilvie and M. Okswa, Phys. Rev. Lett. 53 (1984) 875.

63) P. Stolorz, Caltech Preprint CALT-68-1323 (1988).

[64] K. H. Mutter and K. Schilling, Nucl. Phys. B230 (FSIUj 1984) 275.

[65] A. Konig, K. H. Mutter and K. Schilling, Nucl. Phys. B250 (1985)33. and in this Conference's Proceedings.

[66] R. Gupte and R. Cordery, Phys. Lett. A108 (1984) 115.

67) R. Gupta in Proceedings of the Tallahassee Conference on Advances in Lattice Gauge Theory, World Scientific (1985).

(68) R. Shankar, R. Gupta and G. Murthy, Phys. Rev, Lett. 55 (1985) 1812.

$69 \mid$ R. Gupta, C. Umrigar and K. G. Wilson, under progress.

(70) R. Shankar, Yale preprint YTP 85-25 Nov. (1965).

71) A. Gockech and M. Ogilvie, Phys. Rev. Lett. 64 (1985) 1885. 